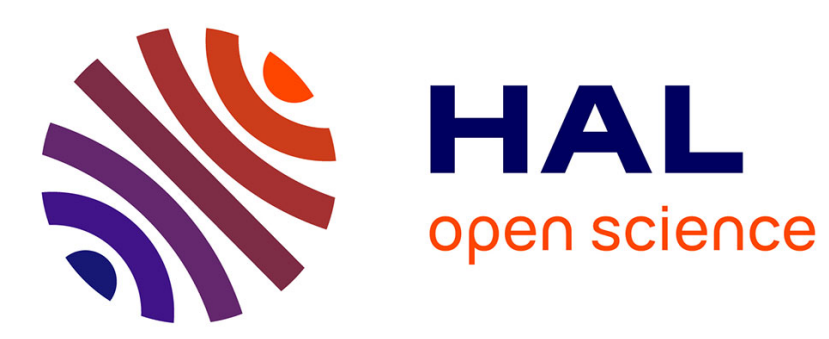

\title{
A Cross-Sectional Performance Measure for Portfolio Management
}

Monica Billio, Ludovic Calès, Dominique Guegan

\section{To cite this version:}

Monica Billio, Ludovic Calès, Dominique Guegan. A Cross-Sectional Performance Measure for Portfolio Management. 2010. halshs-00523466

\section{HAL Id: halshs-00523466 \\ https://shs.hal.science/halshs-00523466}

Submitted on 5 Oct 2010

HAL is a multi-disciplinary open access archive for the deposit and dissemination of scientific research documents, whether they are published or not. The documents may come from teaching and research institutions in France or abroad, or from public or private research centers.
L'archive ouverte pluridisciplinaire HAL, est destinée au dépôt et à la diffusion de documents scientifiques de niveau recherche, publiés ou non, émanant des établissements d'enseignement et de recherche français ou étrangers, des laboratoires publics ou privés. 


\section{Documents de Travail du Centre d'Economie de la Sorbonne}
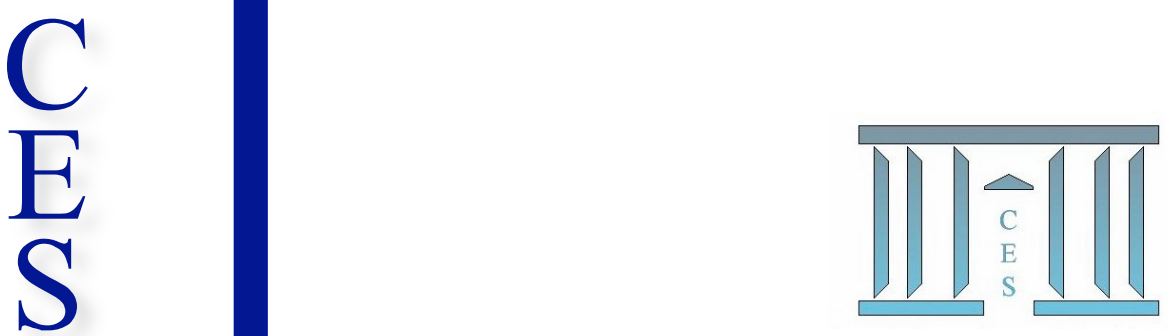

A Cross-Sectional Performance Measure for Portfolio Management

Monica BILLIO, Ludovic CALÈs, Dominique GUEGAN

2010.70

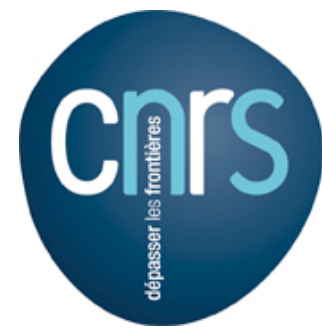




\title{
A Cross-Sectional Performance Measure for Portfolio Management
}

\author{
Monica BILLIO, Ludovic CALÈS and Dominique GUEGAN
}

August 10, 2010

\begin{abstract}
Sharpe-like ratios have been traditionally used to measure the performances of portfolio managers. However, they are known to suffer major drawbacks. Among them, two are intricate: (1) they are relative to a peer's performance and (2) the best score is generally assumed to correspond to a "good" portfolio allocation, with no guarantee on the goodness of this allocation. Last but not least (3) these measures suffer significant estimation errors leading to the inability to distinguish two managers' performances. In this paper, we propose a cross-sectional measure of portfolio performance dealing with these three issues. First, we define the score of a portfolio over a single period as the percentage of investable portfolios outperformed by this portfolio. This score quantifies the goodness of the allocation remedying drawbacks (1) and (2). The new information brought by the cross-sectionality of this score is then discussed through applications. Secondly, we build a performance index, as the average cross-section score over successive periods, whose estimation partially answers drawback (3). In order to assess its informativeness and using empirical data, we compare its forecasts with those of the Sharpe and Sortino ratios. The results show that our measure is the most robust and informative. It validates the utility of such cross-sectional performance measure.
\end{abstract}

Keywords: Performance Measure, Portfolio Management, Relative-Value Strategy, Large Portfolios, Absolute Return Strategy, Multivariate Statistics, Generalized Hyperbolic Distribution

Early versions of this paper have been presented in an invited session at the Computational and Financial Econometrics conference in Limassol, Cyprus, in October 2009, and at the XI Workshop on Quantitative Finance in Palermo, Italy, in January 2010. We thank Marco Nicolosi and the participants of the conferences for their comments and feedback. The authors are also grateful to Stephen Brown for his very helpful comments. 


\section{Introduction}

In this paper, we propose a cross-sectional measure of portfolio performance which deals with three issues encountered by its Sharpe-like ratio counterparts: (1) Sharpe-like ratios are relative to a peer's performance, $(2)$ the portfolio providing the best score is generally assumed to correspond to a "good" allocation, with no guarantee on the goodness of this allocation and (3) these measures suffer significant estimation errors leading to the inability to distinguish two managers' performances.

During the last two decades, the explosive growth of the asset management industry came with an increasing interest in the analysis of investment performance. The research in this area is axed on Sharpe-like ratios proposed in the 60's [Sharpe (1966), Treynor (1965), Jensen (1968)] and it is expanding by developing the notion of performance as a reward counter-balanced by some risk. The main innovations focused on the definition and modeling of risk [Shadwick and Keating (2002), Darolles et al. (2009)]. Practically, the performance of a portfolio manager, over a given period, is usually computed as the ratio of his excess return over a risk measure [Grinblatt et al. (1994)]. The managers are then ranked according to these ratios, and the manager providing the highest and steadiest returns receives the best score. These measures are convenient because they require no assumption on the strategy of the portfolio managers. However, they suffer major drawbacks. First, these measures are relative to a peer's performance and irrelevant if no peer is found. We generally assume that the best score corresponds to a "good" portfolio allocation, with no guarantee on the goodness of this allocation. Secondly, as they are a ratio of two random variables, they suffer significant estimation errors [Lo (2002) and Christie (2007) among others] which prevent any performance comparison to be significant. In this paper, we propose a new measure based on cross-section which deals these issues.

First, we introduce a score which quantifies the quality of an allocation over a single period. For a given investment strategy, this score provides the percentage of investable portfolios which are outperformed by the portfolio in consideration. By construction this score is independent of the period considered. In addition, for a given set of investable portfolio defined by the investment strategy, it reflects the goodness in the choice of an allocation. Thus, it quantifies the ability of the portfolio manager to choose his portfolio. At the authors' knowledge, such a score is new in the literature despite its intuitiveness. This may be due to the difficulty to consider the set of the investable portfolios which is a combinatorial problem.

Secondly, we study in details the computation and the properties of the score for the Zero-Dollar Long/Short Equally Weighted (LSEW) strategy especially in the case of a very large number of assets. Dealing with combinatorial issue inherent to the enumeration of the investable portfolios, 
we introduce an exchangeability assumption in the asset returns. The validity of this assumption is verified empirically in Section III. In the general framework of returns characterized by generalized hyperbolic distributions, as in Eberlein et al. (1995) and Prause (1999), we show that the score is independent of the mean, the variance and the covariance of the returns. In other words, it depends only on the shape parameters of the distribution and justifies to work with this class of distributions. Then, through examples, we show how this score can be used to control one's allocation.

Thirdly, this score is designed for an allocation over a single period while Sharpe-like ratios deal with the performance of investment methods over multiple periods. So, in order to assess the informativeness of our score, we build a performance measure as the average score over a sample. As stated in Sharpe (1994), "most performance measures are computed using historic data but justified on the basis of predicted relationship". So, we motivate our measure by showing through empirical implementations that its predictive ability is superior to those of the other main performance measures, based on Sharpe and Sortino ratios. The results show that our measure is more robust and more informative that the classical Sharpe and Sortino indices. Our score being cross-sectional, these results are supporting the use of cross-sectional data in the design of optimal portfolios as suggested in Brandt et al. (2009).

The paper is organized as follows. In Section II, we introduce the score which quantifies the quality of an allocation over a given period. In Section III, we study and compute this score under fair assumptions. Considering the LSEW investment strategy and assuming that the returns are characterized by generalized hyperbolic distributions, we detail the influence of the distribution parameters on the score. Section IV is devoted to three applications of this score. The first one investigates the relevance of the assumptions. The second one shows how to close the positions of a LSEW portfolio using the methodology developed in the previous sections. The last one monitors a LSEW portfolio in real time. In Section V, we build a performance index based on our score. We show through examples that the choice of portfolio obtained using our index outperforms those using the Sharpe and Sortino ratios. Section VI concludes.

\section{A Cross-Sectional Score of Portfolio Performance}

In this section, we introduce a score quantifying the quality of an allocation. This is achieved by differentiating three elements affecting the performance of a manager: the set of investable portfolios offered to this manager, the period of interest and the allocation method in use. Then, by fixing the two first elements, we aim to measure the performance of an allocation method. First, let focus on the set of investable portfolios. It is usually defined through a set of constraints on the portfolio positions which are stated by the investment policy of the manager. For instance, such constraints might be introduced by short-sale or diversification restrictions. Secondly, over 
two different periods, the use of the returns in order to compare the performance of two managers is delicate. Indeed, two managers using the same allocation method would provide different returns while they are strictly equally performant. In the following, we propose an alternative way to deal with this issue. Finally, the performance of an allocation method should only reflect the correctness of an allocation choice. It is the subject of this section and we aim to assert it by fixing the first two elements.

Let introduce our score. Consider a portfolio manager whose investment policy defines a finite set of portfolios. To provide an objective measure of his allocation performance, we compare the return of his portfolio with the returns of all other investable portfolios. If his portfolio outperforms $S \%$ of all portfolios, we say that it scores $S, S \in[0,1]$. This score $S$ will be the measure of the manager performance that we investigate in details. Because each investable portfolio has a score, the score is cross-sectional over this set. Moreover, this score is interesting because it is independent of the period considered, and thus of the market conditions. In addition, because the set of portfolios is already defined and the period identical to all portfolios, it focuses only on the goodness of the allocation choice. Remark that the score does not need to be compared to a peer's portfolio performance.

Let formalize our score. Denote $\Gamma$ the set of the investable portfolios $\gamma$ induced by the manager's strategy. In a market of $n$ assets, we represent a portfolio as a vector of weights, i.e. $\gamma=(\gamma(1), \ldots, \gamma(n))^{\prime}$ where $\gamma(i)$ is the weight associated with asset $i, i=1, \ldots, n$. In practice, the assets are indivisible and any endowment is finite, so the number of investable portfolios is finite. Moreover, in this paper, we focus on the case where $\Gamma$ is a closed set under taking additive inverses. Such a proceeding leads to a set of portfolios which enables a manager to bet on a market direction as well as on its inverse. This feature is typical of absolute return funds which invest in long and short positions to adapt any market condition. In addition, this proceeding provides a simple interpretation of the score as a score of $50 \%$ corresponds to a return of $0 \%$ and a portfolio with a score $S_{1}$ has an inverse portfolio with a score $1-S_{1}$. We propose now a way to compute the performance measure $S$. Given an invested portfolio $\gamma \in \Gamma$, if $N(\gamma)$ is the number of portfolios outperformed by $\gamma$, then the performance $S$ associated with $\gamma$ is

$$
S(\gamma)=\frac{N(\gamma)}{|\Gamma|}
$$

The computation of $S(\gamma)$ requires the identification of all investable portfolios outperformed by $\gamma$. As soon as $|\Gamma|$ is large, this computation is not direct. To deal with this issue, we introduce the relevant theoretical framework. 
We consider a market of $n$ assets whose returns $\mathbf{X}=\left(X_{1}, \ldots, X_{n}\right)^{\prime}$ have the joint density $f$ and where $Y^{\prime}$ is the transpose of $Y$. The marginal density of $X_{i}, i \in\{1, \ldots, n\}$ is denoted $f_{i}$, and the vector of order statistics induced by $\mathbf{X}$ is $\mathbf{X}_{(\mathbf{n})}=\left(X_{(1)}, X_{(2)}, \ldots, X_{(n)}\right)^{\prime}$. Let be a portfolio $\gamma \in \Gamma$, it returns $\gamma^{\prime} \mathbf{X}$, then for any realization $\mathbf{x}=\left(x_{1}, x_{2}, \ldots, x_{n}\right)^{\prime}, \mathbf{x}_{(n)}$ being a permutation of the elements of $\mathbf{x}$, it exists a portfolio $\tilde{\gamma} \in \Gamma$ such that

$$
\gamma^{\prime} \mathbf{x}=\tilde{\gamma}^{\prime} \mathbf{x}_{(\mathbf{n})}
$$

In the following, we denote $g$ the density of $\gamma^{\prime} \mathbf{X}$ and $u_{\tilde{\gamma}}$ the density of $\tilde{\gamma}^{\prime} \mathbf{X}_{(\mathbf{n})}$. As $\Gamma$ is finite, there exists an optimal portfolio $\gamma_{o}$ which provides the highest return for a given realization $\mathbf{x}$. Its order statistic is denoted $\tilde{\gamma}_{o}$ and its return is equal to

$$
\gamma_{o}^{\prime} \mathbf{x}=\tilde{\gamma}_{o}^{\prime} \mathbf{x}_{(\mathbf{n})}
$$

It is helpful to remark that the return of any portfolio $\gamma \in \Gamma$ can be expressed relatively to the return of the optimal portfolio $\tilde{\gamma}_{o}$. This means that there exists a parameter $k \in[-1,1]$ such that:

$$
\gamma^{\prime} \mathbf{x}=k \tilde{\gamma}_{o}^{\prime} \mathbf{x}_{(\mathbf{n})}
$$

By definition, the optimal portfolio $\gamma_{o}$ scores $S=1$ and its opposite - which is the worst portfolio - scores $S=0$.

Back to the computation of $S$, we use the parameter $k$ introduced in (3) which can be associated to any portfolio $\gamma \in \Gamma$. Thus, to obtain an approximation of $S\left(\gamma_{i}\right)$ for a given portfolio $\gamma_{i}$, we approximate the number of portfolios $N\left(\gamma_{i}\right)$ by the expected number of portfolios returning less than $k_{i}$ times the return of the optimal portfolio. We denote this expected number $\bar{N}\left(k_{i}\right)$ :

$$
\begin{aligned}
\bar{N}\left(k_{i}\right) & =E\left(\left|\left\{\gamma \in \Gamma \mid \gamma^{\prime} \mathbf{X} \leq k_{i} \tilde{\gamma}_{o}^{\prime} \mathbf{X}_{(\mathbf{n})}\right\}\right|\right) \\
& =\sum_{\gamma \in \Gamma} P\left(\gamma^{\prime} \mathbf{X} \leq k_{i} \tilde{\gamma}_{o}^{\prime} \mathbf{X}_{(\mathbf{n})}\right) \\
& =\sum_{\gamma \in \Gamma}\left(\sum_{\tilde{\gamma} \in \Gamma} P\left(\tilde{\gamma}^{\prime} \mathbf{X}_{(\mathbf{n})} \leq k_{i} \tilde{\gamma}_{o}^{\prime} \mathbf{X}_{(\mathbf{n})}\right) P\left(\gamma^{\prime} \mathbf{X}=\tilde{\gamma}^{\prime} \mathbf{X}_{(\mathbf{n})}\right)\right) \\
& =\left(\sum_{\tilde{\gamma} \in \Gamma} P\left(\tilde{\gamma}^{\prime} \mathbf{X}_{(\mathbf{n})} \leq k_{i} \tilde{\gamma}_{o}^{\prime} \mathbf{X}_{(\mathbf{n})}\right)\right)\left(\sum_{\gamma \in \Gamma} P\left(\gamma^{\prime} \mathbf{X}=\tilde{\gamma}^{\prime} \mathbf{X}_{(\mathbf{n})}\right)\right) .
\end{aligned}
$$


Observing that $\sum_{\gamma \in \Gamma} P\left(\gamma^{\prime} \mathbf{X}=\tilde{\gamma}^{\prime} \mathbf{X}_{(\mathbf{n})}\right)=1$, we obtain

$$
\begin{aligned}
\bar{N}\left(k_{i}\right)=\sum_{\tilde{\gamma} \in \Gamma} P\left(\tilde{\gamma}^{\prime} \mathbf{X}_{(\mathbf{n})} \leq k_{i} \tilde{\gamma}_{o}^{\prime} \mathbf{X}_{(\mathbf{n})}\right) & =\sum_{\tilde{\gamma} \in \Gamma} P\left(\left(\tilde{\gamma}^{\prime}-k_{i} \tilde{\gamma}_{o}^{\prime}\right) \mathbf{X}_{(\mathbf{n})} \leq 0\right) \\
& =\sum_{\gamma \in \Gamma} P\left(\left(\gamma^{\prime}-k_{i} \tilde{\gamma}_{o}^{\prime}\right) \mathbf{X}_{(\mathbf{n})} \leq 0\right)
\end{aligned}
$$

If we denote $f_{\gamma, k_{i}}$ the density of $\left(\gamma^{\prime}-k_{i} \tilde{\gamma}_{o}{ }^{\prime}\right) \mathbf{X}_{(\mathbf{n})}$, the relationship (5) becomes:

$$
\bar{N}\left(k_{i}\right)=\sum_{\gamma \in \Gamma} \int_{-\infty}^{0} f_{\gamma, k_{i}}(y) d y
$$

Plugging relationship (6) in equation (1) provides an approximation of the score for any portfolio $\gamma_{i}$ returning $k_{i}$ times the return of the optimal portfolio $\gamma_{o}$ :

$$
\bar{S}\left(k_{i}\right)=\frac{\bar{N}\left(k_{i}\right)}{|\Gamma|}=\frac{1}{|\Gamma|} \sum_{\gamma \in \Gamma} \int_{-\infty}^{0} f_{\gamma, k_{i}}(y) d y .
$$

As soon as the number of assets is large, the enumeration of the portfolios of $\Gamma$ is laborious and the computation of (7) remains difficult. To achieve this computation, we introduce a technical assumption on the returns and provide the resulting expression of $\bar{S}$ in the next proposition.

$A_{0}:$ The asset returns are exchangeable.

We recall that a sequence of random variables is exchangeable if, for any permutation of these random variables, the joint probability distribution of the rearranged sequence is the same as the joint probability of the original sequence, Arellano-Valle and Genton (2007). In particular, a sequence of independent and identically distributed (i.i.d.) random variables is exchangeable. The validity of assumption $\left(A_{0}\right)$ is verified empirically in Section IV-A.

Proposition 1: Let $\mathbf{X}$ be an exchangeable random vector. Denote $\mathbf{X}_{(\mathbf{n})}$ its corresponding vector of order statistics, $\gamma_{o}$ the optimal portfolio and $\gamma$ a portfolio returning $k$ times the return of $\gamma_{o}$, then the approximated score $\bar{S}$ for a portfolio $\gamma$ is equal to

$$
\bar{S}(k)=\int_{-\infty}^{0}\left(g * h_{k}\right)(y) d y
$$


where $*$ stands for the convolution product; $g$ is the density of $\gamma^{\prime} \mathbf{X}$ and $h_{k}$ the density of $-k \tilde{\gamma}_{o}^{\prime} \mathbf{X}_{(\mathbf{n})}$, where $\tilde{\gamma}_{o}$ is the ordered representation of the optimal portfolio $\gamma_{o}$.

Proof: The proof of this proposition is postponed in Appendix B.

\section{Estimation of the Score}

In this section, we thoroughly study the score $S$ in the case of the zero-dollar long/short equally weighted strategy (LSEW). Assuming the generic case where the assets' returns follow a multivariate generalized hyperbolic distribution, we investigate the effects of the distributions parameters on the score. Next, we illustrate the cases of the 130/30 and Hamming strategies.

\section{A The case of the LSEW Strategy}

First of all, let introduce the LSEW strategy. This strategy consists in investing in portfolios which are long/short (i.e. include both long and short positions), zero-dollar (the value of the long positions is equal to the value of the short positions) and equally weighted (each position has the same value in absolute value). In addition, the leverage of these portfolios is fixed to $2: 1$. The notation 2:1 means that the amount of capital backing the portfolio represents $50 \%$ of the portfolio value. It is the minimum amount required under the U.S. Regulation (namely Regulation T). As a consequence, in our case, the sum of the absolute values of the weights of the portfolio equals 2 . The LSEW strategy is particularly interesting because it is the one used to track the momentum effect in most of the literature [Jeegadeesh and Titman (1993), Rouwenhorst (1998), Chan et al. (2000), Okunev and White (2003), Kazemi et al. (2009) and Billio et al. (2009) among others]. This LSEW strategy is also the base of most of the relative value strategies (arbitrage) which take advantage of the mispricing between two assets [see Gatev et al. (1999) for the case of pair trading]. Note that the consideration of equal weights is not as limiting as it seems in the choice of the portfolio. On the contrary, as shown in Demiguel et al. (2009), the errors in estimating the means and covariances of the assets' returns penalize the mean-variance optimization enough to erase the diversification gain and provide portfolios with a lower out-of-sample Sharpe ratio than the naive equally weighted portfolio.

For instance, in a market of 4 assets $(A, B, C, D)$, there are 6 LSEW portfolios. We represent them in Table 1. Note that there are $|\Gamma|=\frac{n !}{\left(\frac{n}{2} !\right)^{2}}$ LSEW portfolios in a market of $n$ assets. So, the number of portfolios increases exponentially with $n$. As an illustration of the combinatorial issue, a market of 30 assets leads to $1.5510^{8}$ portfolios which would require 4.33 Go of memory to be stocked and prevent any enumeration. 


\begin{tabular}{|r|r|r|r|r|r|r|}
\hline & $\gamma_{1}$ & $\gamma_{2}$ & $\gamma_{3}$ & $\gamma_{4}$ & $\gamma_{5}$ & $\gamma_{6}$ \\
\hline Asset A & $1 / 2$ & $-1 / 2$ & $1 / 2$ & $-1 / 2$ & $1 / 2$ & $-1 / 2$ \\
\hline Asset B & $1 / 2$ & $1 / 2$ & $-1 / 2$ & $1 / 2$ & $-1 / 2$ & $-1 / 2$ \\
\hline Asset C & $-1 / 2$ & $-1 / 2$ & $-1 / 2$ & $1 / 2$ & $-1 / 2$ & $1 / 2$ \\
\hline Asset D & $-1 / 2$ & $1 / 2$ & $1 / 2$ & $-1 / 2$ & $1 / 2$ & $1 / 2$ \\
\hline
\end{tabular}

Table 1: The set $\left\{\gamma_{1}, \gamma_{2}, \gamma_{3}, \gamma_{4}, \gamma_{5}, \gamma_{6}\right\}$ is the set of the LSEW portfolios that can be built in a market of 4 assets, here $\{A, B, C, D\}$.

In addition, the optimal portfolio is then long the $\frac{n}{2}$ assets which perform the best and is short the $\frac{n}{2}$ assets which perform the worst:

$$
\tilde{\gamma}_{o}(i)=\left\{\begin{array}{rrr}
-2 / n & , \text { if } & i \leq n / 2 \\
2 / n & \text {, if } & i>n / 2
\end{array}\right.
$$

In practice, the computation of $\bar{S}$ using the expression (8) requires to determine the density $g$ corresponding to a linear combination of $n$ random variables, the density $h_{k}$ corresponding to the linear combination of $n$ order statistics and the convolution product between $g$ and $h_{k}$. For the computation of $h_{k}$, we use the methodology developed by Arellano-Valle and Genton (2007). Nevertheless, their result is difficult to apply as soon as $n$ is large. In that case Monte Carlo simulations are appropriate. Through an example, we carry out the computation of $\bar{S}$.

Let consider a simulated market of 10 assets - inducing $|\Gamma|=252$ LSEW portfolios - whose returns are i.i.d., and follow a Gaussian distribution with mean 0 and variance 0.01. Then, the density $g$ is the sum of 10 independent Gaussian densities, and we calculate the density $h_{k}$ using Monte Carlo simulations, computing $\gamma^{\prime} \mathbf{x}-k \tilde{\gamma}_{o}^{\prime} \mathbf{x}_{(\mathbf{n})}$ for each realization $\mathbf{x}$, with $\gamma \in \Gamma$ and $\tilde{\gamma}_{o}$ the optimal portfolio obtained by ranking the 10 returns. In Figure 2, we represent $\bar{S}$ as a function of $k$. We remark that the score $\bar{S}$ of a portfolio $\gamma_{i}$ providing $k_{i}=60 \%$ of the return of the optimal portfolio is $\bar{S}=92 \%$. This means that only $8 \%$ of the LSEW portfolios provide an higher return than $\gamma_{i}$, on average.

Financial asset returns are well known to have distributions which are asymmetric and leptokurtic. Thus, it is important to be able to compute $\bar{S}$ when the asset returns are modeled by distributions more complex than the Gaussian one. As shown in Eberlein et al. (1995), Prause (1999) and Fajardo et al. (2009), among others, a multivariate generalized hyperbolic distribution can be considered due to its flexibility and its good fitting for financial asset returns. We exhibit such an example in Section IV-A showing the superiority of the fit obtained with the generalized hyperbolic distribution over the Gaussian distribution. Thus, in the following, we assume that the observations $\mathbf{X}=\left(X_{1}, \ldots, X_{n}\right)$ are characterized by a multivariate generalized hyperbolic distribution and we identify the distribution's parameters which affect $\bar{S}$. 


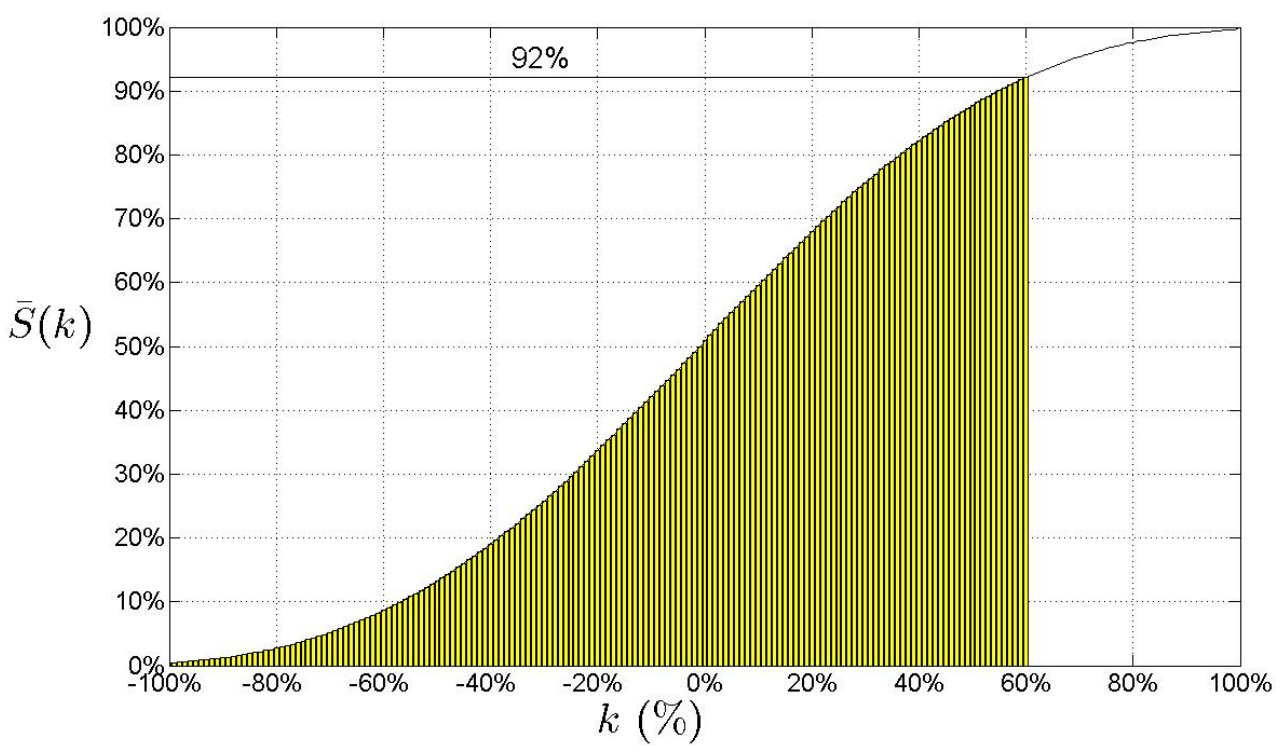

Figure 1: Representation of $\bar{S}$ with respect to $k$, the approximation of the score of an LSEW portfolio whose return is $k$ times the return of the optimal portfolio, as introduced in (8), in a simulated market of 10 assets whose returns are independent and identically distributed according to a Gaussian distribution with mean 0 and standard deviation $1 \%$. We recall that, by definition, the optimal portfolio scores 1 and the worst portfolio scores 0 . In this example, the approximated score of a portfolio returning $60 \%$ of the return of the optimal portfolio is $92 \%$, i.e. with such a return this portfolio outperforms in average $92 \%$ of the LSEW portfolios.

A multivariate generalized hyperbolic distributions $G H_{n}(\lambda, \chi, \psi, \mu, \Sigma, \kappa)$ can be represented as a normal mean-variance mixture [Barndorff-Nielsen et al. (1982)], and is characterized by six parameters: the mean $\mu \in R^{d}$, the variance-covariance matrix $\Sigma \in R^{d \times d}$, the skewness parameter, $\kappa \in R^{d}$, and the shape parameters $\lambda, \chi$ and $\psi$. In the following, we use this very flexible class of distributions to characterize the assets on a market since it contains a lot of well known distributions (Laplace, Student-t, normal inverse Gaussian, inverse Gaussian, etc.). We introduce now a new assumption which permits to extend the results of the Proposition 1.

$A_{1}$ : The asset returns are characterized by a multivariate generalized hyperbolic distribution $G H_{n}(\lambda, \chi, \psi, \mu, \Sigma, \kappa)$.

Under the assumptions $\left(A_{0}\right)$ and $\left(A_{1}\right)$, the vector $\mathbf{X}$ is an exchangeable random vector characterized by a multivariate generalized hyperbolic distribution, and $\Sigma=\sigma^{2}\left[(1-\rho) I_{n}+\rho \mathbf{1}_{\mathbf{n}} \mathbf{1}_{\mathbf{n}}{ }^{\prime}\right]$ where $\sigma$ is the variance of $\mathbf{X}$ and $\rho$ is the correlation between $X_{i}$ and $X_{j}, i, j \in\{1, \ldots, n\}$, [Arellano-Valle and Genton (2007)]. 
Proposition 2: Let $\mathbf{X}$ be an exchangeable random vector distributed according to a multivariate generalized hyperbolic distribution $G H_{n}(\lambda, \chi, \psi, \mu, \Sigma, \kappa), \mathbf{X}_{(\mathbf{n})}$ being the random vector of its order statistics and $\gamma_{o}$ the optimal portfolio, then

$$
\bar{S}(k)=\int_{-\infty}^{0} v_{k}(y) d y
$$

with $v_{k}$ the density function of $Z-k{\tilde{\gamma_{o}}}^{\prime} \mathbf{U}_{(\mathbf{n})}$ where $Z$ is an elliptically contoured random variable $E C_{1}\left(0, \frac{4}{n}, \phi^{(1)}\right)$, and $\mathbf{U}_{(\mathbf{n})}$ is the vector of order statistics induced by $\mathbf{U}_{\mathbf{n}} \sim E C_{n}\left(\mathbf{0}, I_{n}, \phi^{(n)}\right)$ with the density generator $\phi^{(m)}$ given by

$$
\phi^{(m)}(u)=C_{m} \frac{K_{\lambda-\frac{m}{2}}(\sqrt{\psi(\chi+u)})}{(\sqrt{\chi+u})^{\frac{m}{2}-\lambda}}
$$

with $C_{m}$ a normalizing constant, and $K_{\nu}$ the modified Bessel function of the third kind.

Proof: The proof of this proposition is postponed in Appendix C.

We remark that the score $\bar{S}$ depends only on the shape parameters $\lambda, \chi$ and $\psi$ which confirms the limitations implied by working in the Gaussian framework. Therefore, in the case of Gaussian i.i.d. returns as presented in Figure 2, different means and different variances and correlations would lead to the same function $\bar{S}(k)$. In Section IV-B, figure 4 , we compare the score $\bar{S}_{\text {Gaussian }}$ obtained with a Gaussian distribution of the returns and the score $\bar{S}_{G H D}$ obtained with a generalized hyperbolic distribution (GHD) fitted on market data. We observed that investing in a market where the returns follow a GHD distribution is slightly more risky than in a market where the returns follow a Gaussian distribution because $\bar{S}_{G H D}(k)>\bar{S}_{\text {Gaussian }}(k)$ for $k<0$. In the other hand, $\bar{S}_{G H D}(k)<\bar{S}_{\text {Gaussian }}(k)$ for $k>0$, so an investment in the GHD market is more likely to provide higher scores than an investment in the Gaussian market.

\section{B The case of the 130/30 and 'Hamming' Strategies}

The score presented in the previous section is used with the LSEW strategy and it can be applied to any other strategy. In this section, we present the score obtained with two other equally weighted strategies: the 130/30 strategy and the Hamming strategy. The 130/30 strategy is long (or short) $130 \%$ of the portfolio and short (or long) 30\%. All the portfolios generated have a leverage of $1.6: 1$. The Hamming strategy is long or short any position without constraint. The weights of the portfolios are adjusted in a way such that they all have a leverage of $2: 1$. In Figure 2 , we represent $\bar{S}$ as a function of $k$ for these two strategies along with the LSEW strategy. 


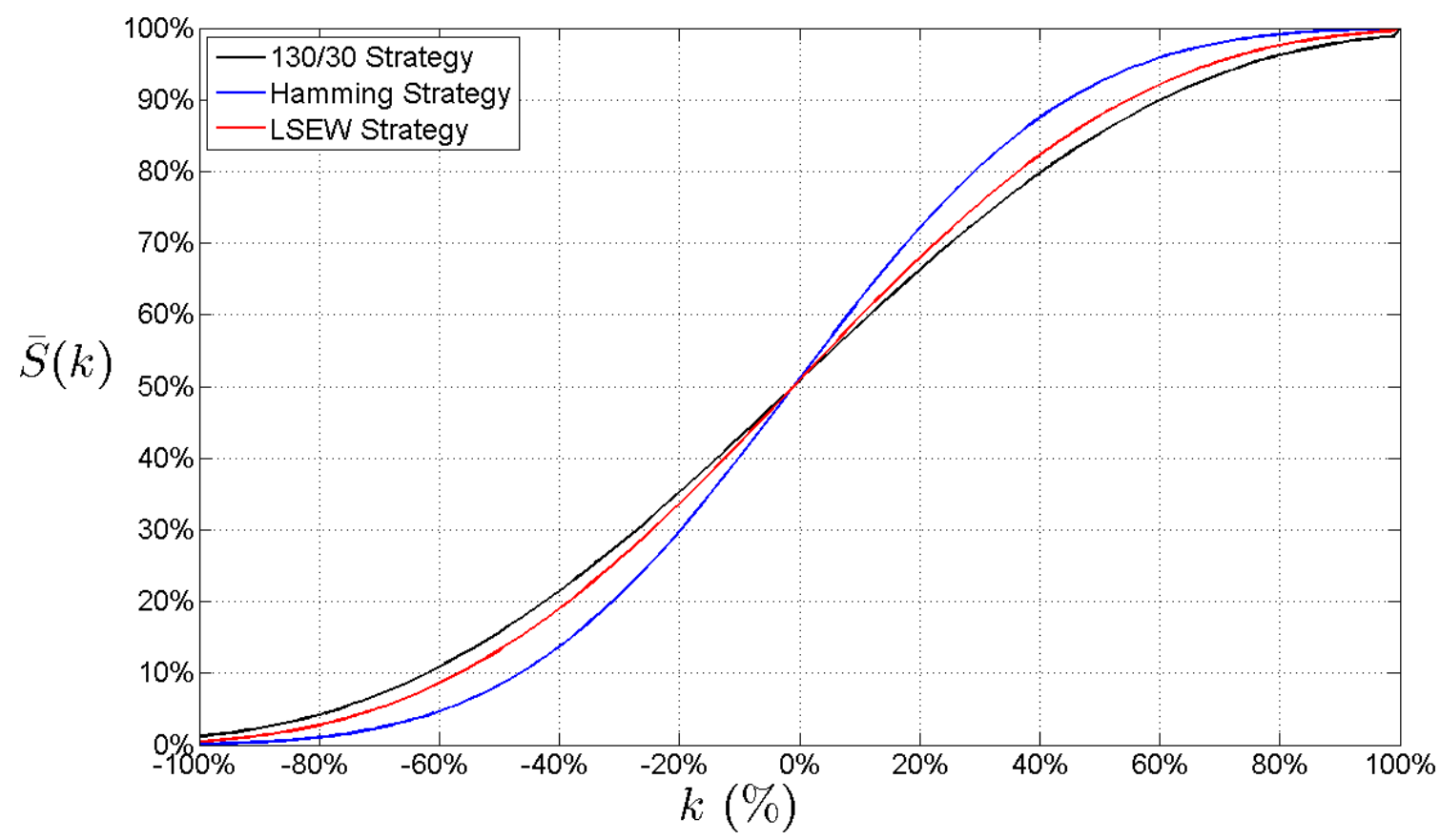

Figure 2: Representation of $\bar{S}$ with respect to $k$, as defined in (8), for three different investment strategies: the LSEW strategy, the 130/30 strategy and the Hamming strategy. For each of these strategy, $\bar{S}(k)$ is the approximation of the score of a portfolio (within the set of the portfolios generated by the strategy) whose return is $k$ times the return of the optimal portfolio, in a simulated market of 10 assets whose returns are independent and identically distributed according to a Gaussian distribution with mean 0 and standard deviation 1\%. By definition, the optimal portfolio scores 1 and the worst portfolio scores 0 .

We observe that:

- for $k<0, \bar{S}_{130 / 30}>\bar{S}_{L S E W}>\bar{S}_{\text {Hamming }}$ : so, by choosing randomly a portfolio, the probability to obtain a low score is higher for the 130/30 strategy, next followed by the LSEW strategy and finally by the Hamming strategy.

- for $k>0, \bar{S}_{\text {Hamming }}>\bar{S}_{L S E W}>\bar{S}_{130 / 30}$ : so, by choosing randomly a portfolio, the probability to obtain a high score is higher for the 130/30 strategy, next followed by the LSEW strategy and finally by the Hamming strategy.

The symmetry of the strategies implies that the probability to get a high score $(k>0)$ by choosing randomly a portfolio is off-set by the probability to get a low score $(k<0)$. An investor who is concerned by the scores of his invested portfolio would prefer the Hamming strategy if he is risk averse and the $130 / 30$ if he is risk lover. 


\section{Applications and Empirical Validation}

We provide now three applications showing the interest of our methodology. The first one investigates the impact of assumptions $\left(A_{0}\right)$ and $\left(A_{1}\right)$; the second one proposes a new exit strategy for managers willing to close their positions and the third one illustrates the usefulness of this measure for monitoring portfolios in real time.

\section{A Empirical relevance of the assumptions $\left(A_{0}\right)$ and $\left(A_{1}\right)$}

Let consider a market whose returns follow an arbitrary random vector $\mathbf{X}$. In order to verify that the assumptions $\left(A_{0}\right)$ and $\left(A_{1}\right)$ are not too strong to be relevant, we compare the score $\bar{S}$ computed assuming $\left(A_{0}\right)$ and $\left(A_{1}\right)$ and the score $\breve{S}(k)$ computed as the average percentage of portfolios returning less than $k$ times the return of the optimal portfolio, using the relationship (1). Practically, to obtain $\breve{S}(k)$, we need to enumerate all the LSEW portfolios. In our example, we restrict ourselves to a market of 10 assets, corresponding to 252 LSEW portfolios. The market is composed by the 10 Datastream ${ }^{\mathrm{TM}}$ sectorial world indices, with their monthly returns, from January 1975 to May 2008. The Datastream ${ }^{\mathrm{TM}}$ codes of the indices are reported in Appendix A. To compute $\bar{S}$, we assume that the asset returns are stationary, exchangeable and characterized by a generalized hyperbolic $(\mathrm{GH})$ distribution. Here, we fit the assets' returns with a Normal Inverse Gaussian (NIG) distribution $(\lambda=-0.5)$. Because the assets returns are assumed to be exchangeable, they all have the same distribution which is estimated through a fit over the concatenation of all the assets returns. Such a proceeding does not allow to estimate the correlation between the assets which in any way is irrelevant to the computation of the score as shown in Proposition 2. The estimation has been performed using the Matlab package developed by Saket Sathe which is available on-line in the Matlab(C)Central web site: http://www.mathworks.com.

In order to illustrate the accuracy of our choice, we propose in Figure 3 the Q-Q plots corresponding to the adjustments of a Gaussian distribution and a NIG one over the distribution of the pooled assets returns. The Q-Q plots clearly show the superiority of the fit obtained using the NIG distribution. In Table 2, we exhibit the p-values of the Kolmogorov-Smirnov test considering our empirical sample of 4010 returns $(10$ (assets) $\times 401$ (months) $)$. Under the null hypothesis, we first assume that the empirical sample is drawn from the Gaussian distribution, and next from the NIG distribution.

\begin{tabular}{|r|r|r|}
\hline & Gaussian dist. & NIG dist. \\
\hline p-value & $2.017510^{-5}$ & 0.7846 \\
\hline
\end{tabular}

Table 2: P-values obtained with the Kolmogorov-Smirnov tests between the empirical distribution of the returns of the 10 Datastream world sectorial indices pooled all together and a fitted Gaussian distribution, and between the same empirical distribution and a fitted NIG distribution. 

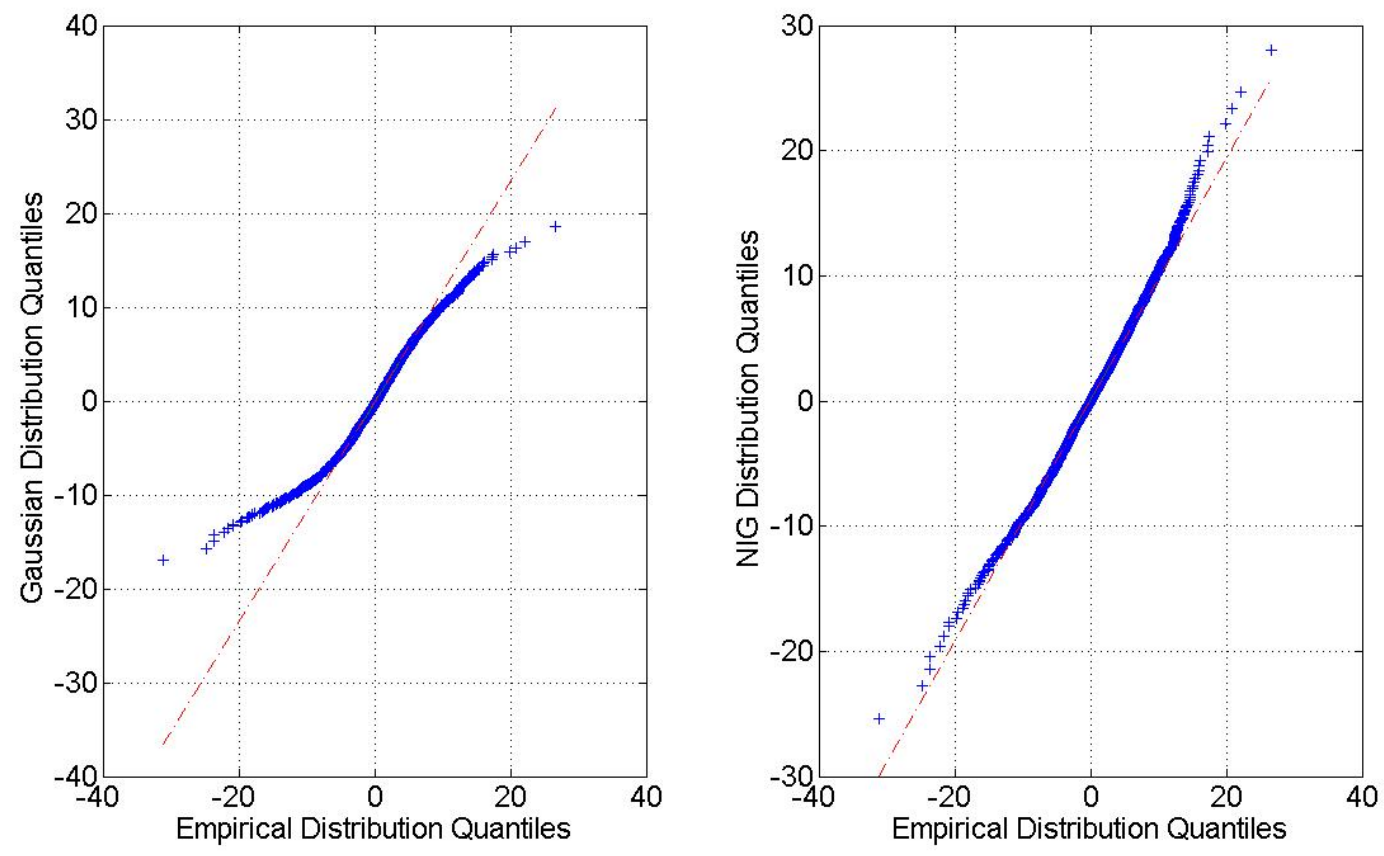

Figure 3: Comparison of the Q-Q plots obtained by plotting the empirical distribution of the returns of the 10 Datastream world sectorial indices pooled all together against a fitted Gaussian distribution (in the left plot) and against a fitted NIG distribution (in the right plot). It shows the superiority of the fit obtained using the NIG distribution.

The test validates the choice of the NIG distribution for the returns ( $p$-value higher that 0.05). In order to illustrate the impact of the distribution's choice for the returns, we provide the scores $\bar{S}$ issued from the Gaussian hypothesis denoted $\bar{S}_{N}$, and from the NIG hypothesis denoted $\bar{S}_{N I G}$. Both scores are computed using Monte Carlo simulations using the 4010 returns. In Figure 4, we represent $\bar{S}_{N I G}$ with the blue line, $\breve{S}$ with the red line, and $\bar{S}_{N}$ with the black dot line.

We observe that $\bar{S}_{N I G}$ and $\breve{S}$ coincide. The blue line covers the red one almost everywhere. Thus, it seems that the assumptions $\left(A_{0}\right)$ and $\left(A_{1}\right)$ used to compute $\bar{S}_{N I G}(k)$ do not create any relevant bias in the computation of the score. When we assume that the data set comes from a Gaussian random vector - which is invalidated in Table 2 - we observe a difference between $\bar{S}_{N}$ (black dashed line) and $\breve{S}$ (red line). The score $\bar{S}_{N}$ underestimates $\breve{S}$ for negative $k$ and overestimates it for positive $k$. Thus, $\bar{S}_{N I G}$ can be considered as a better approximation of $\breve{S}$ than $\bar{S}_{N}$. 


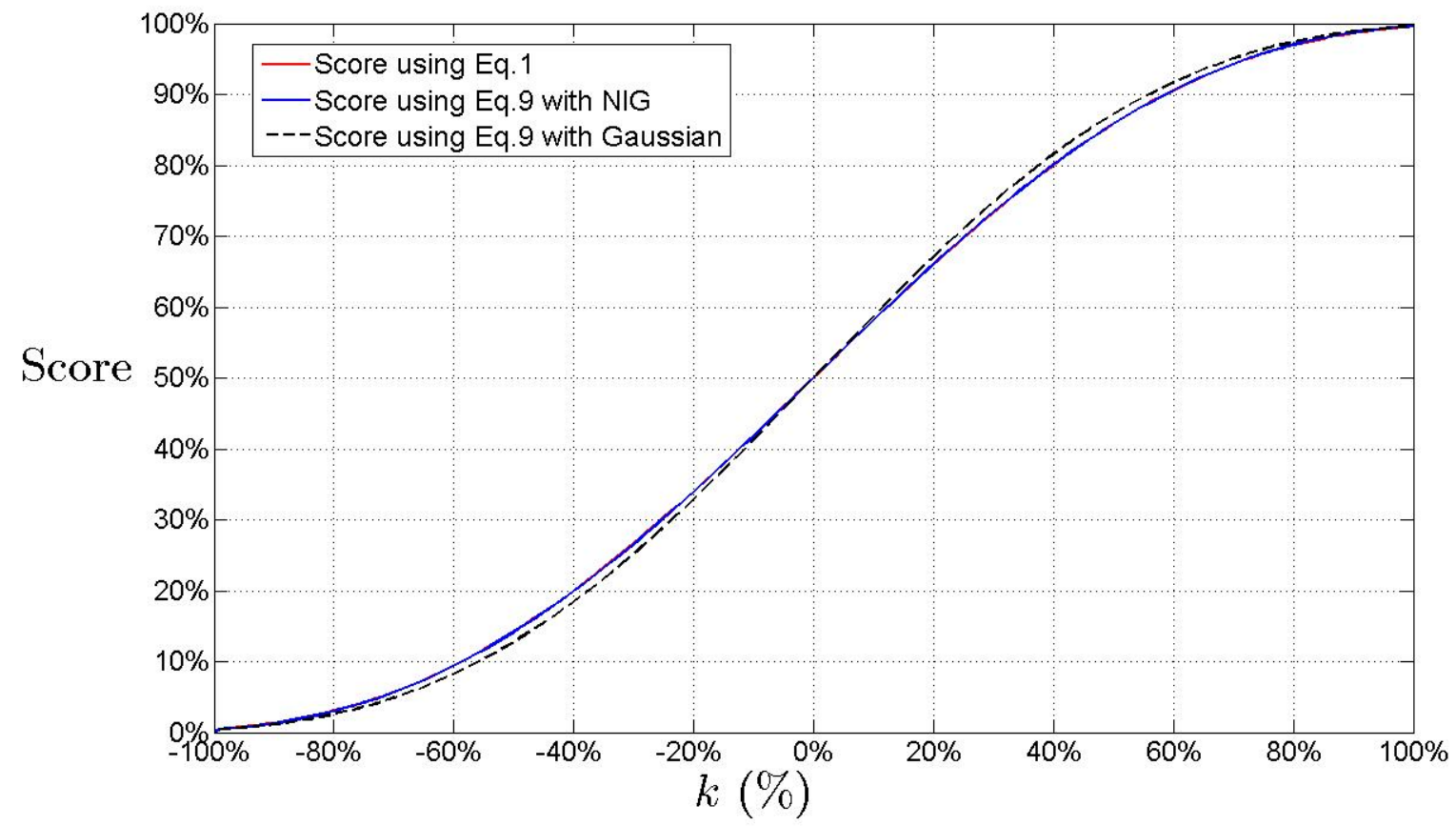

Figure 4: Representations of $\breve{S}(k), \bar{S}_{N I G}(k)$ and $\bar{S}_{N}(k)$, three different approximations of the score of an LSEW portfolio whose return is $k$ times the return of the optimal portfolio, in the market of the 10 Datastream world sectorial indices. $\breve{S}(k)$ is the average percentage of portfolios returning less than $k$ times the return of the optimal portfolio as observed in the market. $\bar{S}_{N I G}$ is the approximated score of a portfolio returning $k$ times the return of the optimal portfolio obtained by fitting a NIG distribution on the returns and assuming $\left(A_{0}\right)$ and $\left(A_{1}\right) . \bar{S}_{N}$ is identical to $\bar{S}_{N I G}$ except for the fitting of the distribution which is Gaussian.

\section{B Application to exit positions}

An interesting application of the score $S$ is to use it to appreciate the opportunity to close positions. Indeed, this new measure quantifies the goodness of an allocation for given market conditions. We consider a manager whose portfolio is invested, and we assume that, due to fluctuating market conditions, the knowledge of his portfolio's return is not sufficient to decide to close his positions. Suppose now that the portfolio provides a high score, $S=90 \%$, then its return is among the highest possible ones for a given time and given market conditions. Roughly speaking, the manager has achieved the most it was possible to perform. Consequently, a reasonable decision is to close the positions and try to do his best over the next period. As an illustration, we consider the following LSEW portfolio $\gamma$ invested on the 10 Datastream ${ }^{\mathrm{TM}}$ world sectorial indices, and in Table 3 we report the weights of this portfolio. 


\begin{tabular}{|ccccc|}
\hline Oil\&Gas & Basic Mat. & Industry & Consumer Gds & Health Care \\
\hline$-0,2$ & $-0,2$ & $-0,2$ & $-0,2$ & 0,2 \\
\hline Consumer Svs & Telecom & Utilities & Finance & Techno \\
\hline 0,2 & $-0,2$ & 0,2 & 0,2 & 0,2 \\
\hline
\end{tabular}

Table 3: Weights of the invested LSEW portfolio $\gamma$ in the 10 sectorial indices. The sum is 2 because the leverage used is $2: 1$.

Suppose that this LSEW portfolio has been invested the 31/07/2009 at the closing time. Then, the portfolio manager can follow the score of his portfolio over the next days. We give the evolution of the score $S(\gamma)$ in Figure 5 from July 31, 2009 to September 30, 2009. We observe that the first days the score of the portfolio is poor: indeed it is starting below $30 \%$. However after few days, it performs particularly well because it is above $80 \%$. Finally, after 30 days it drops to the median score (around 50\%). Note that thanks to the symmetry of the LSEW strategy a score below $50 \%$ corresponds to a negative return and respectively a score above $50 \%$ corresponds to a positive return. The example shows that it would have been timely for the manager to close his positions between the 15th of August and the 04th of September.

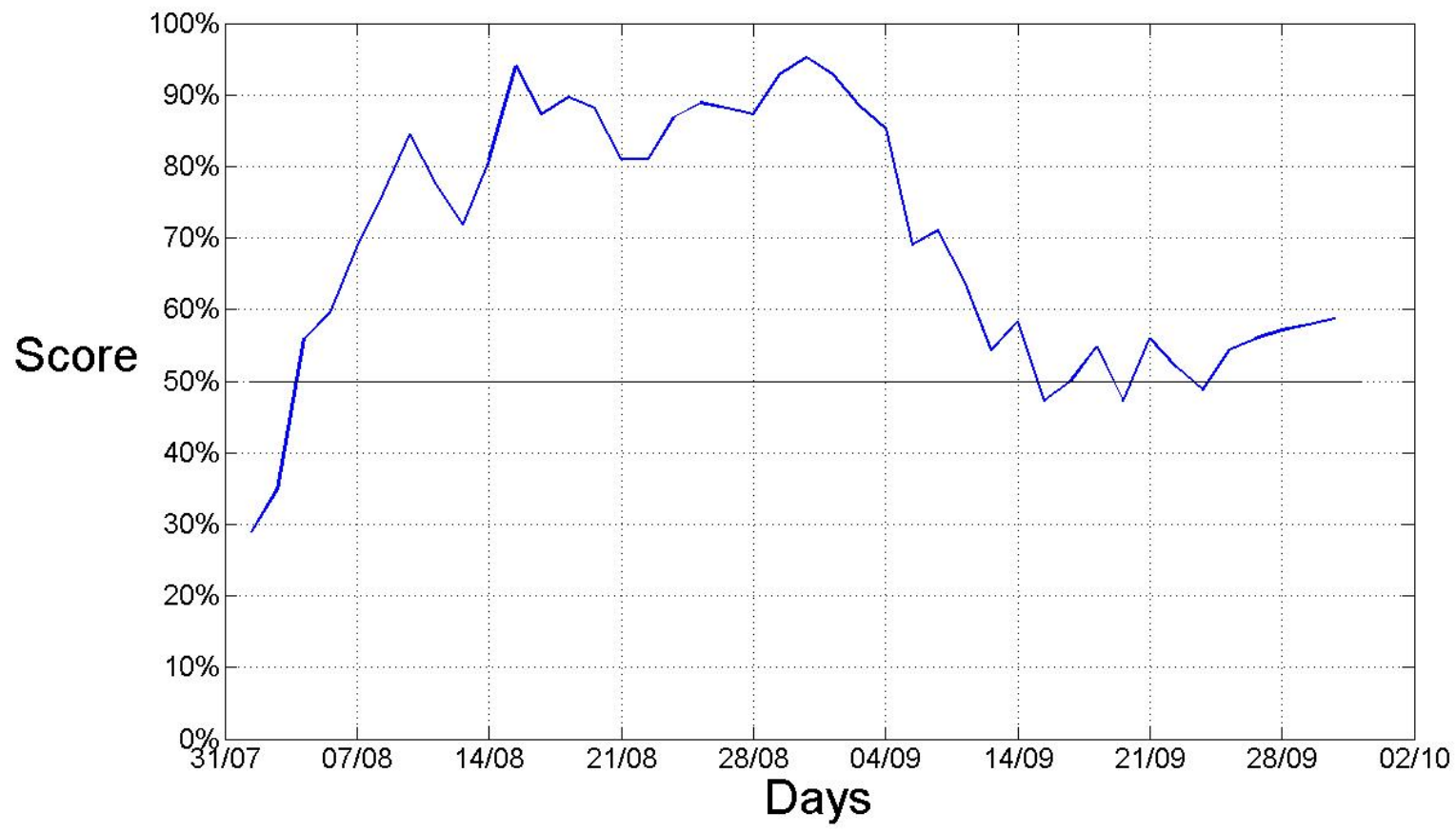

Figure 5: Observed score of the invested LSEW portfolio defined in Table 3 from the 31/07/2009 at the closing and over the 42 following days. This score is obtained by enumeration over all the LSEW portfolios and corresponds to the real score of the invested portfolio. 


\section{Monitoring with the Estimated Score}

Given a portfolio $\gamma$ invested at date $t=0$ we compare its scores $\left(\bar{S}_{t}\right)_{t>0}$ defined in $(9)$ with its scores $\left(S_{t}\right)_{t>0}$ defined in (1) at the dates $t \geq 1$. We assume that the assets' log-returns are governed by a strictly stationary process $\left(\mathbf{X}_{\mathbf{t}}\right)_{t>0}$ characterized by a NIG distribution. Given the realizations $\left(\mathbf{x}_{\mathbf{t}}\right)_{t>0}$, the $\log$ return of the portfolio $\gamma$ is approximatively $\gamma^{\prime}\left(\sum_{i \in\{1, ., t\}, t \geq 1} \mathbf{x}_{\mathbf{i}}\right)$ at each time $t$. From relationship (3) we compute the sequence $\left(k_{t}\right)_{t>0}$ associated with $\gamma$ at the dates $t, t>0$. The stationarity property implies that $\bar{S}_{t}\left(k_{t}\right)=\bar{S}\left(k_{t}\right)$ for all t. Therefore, the scores $\left(\bar{S}\left(k_{t}\right)\right)_{t>0}$ derive from the previous sub-sections and the scores $\left(S_{t}\right)_{t>0}$ are directly computed as the percentage numbers of outperformed portfolios for the given realizations. To illustrate our purpose, we use the same portfolio and data set introduced in Sub-section IV-B. The values of $\left(\bar{S}\left(k_{t}\right)\right)_{t>0}$ and $\left(S_{t}\right)_{t>0}$ are reported in Figure 6. We observe that $\bar{S}$ correctly fits $S$.

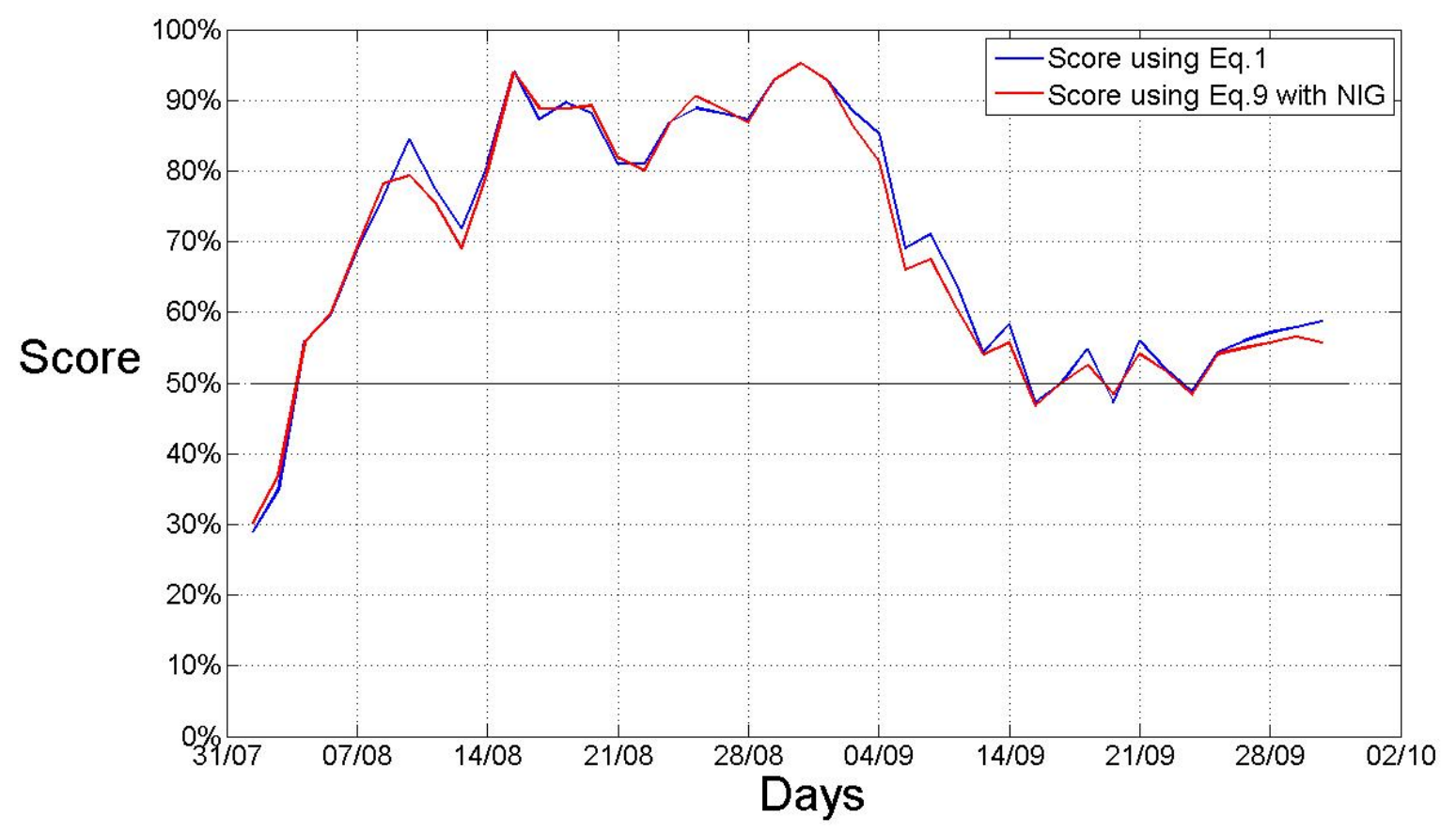

Figure 6: Observed score $S_{t}$ and approximated score $\bar{S}_{t}$ of the invested LSEW portfolio defined in Table 3 from the 31/07/2009 at the closing and over the 42 following days. The score $S_{t}$ is obtained by enumeration over all the LSEW portfolios and thus is the real score of the invested portfolio. $\bar{S}_{t}$ is the approximated score of the invested portfolio obtained by fitting with a NIG distribution and assuming $\left(A_{0}\right)$ and $\left(A_{1}\right)$.

Now, we provide a quantitative criteria for the estimation of $S$ based on the mean absolute error, computing the errors produced by $\bar{S}$ and $\breve{S}$. As $\bar{S}(k)$ is invariant over time, we focus on one-step daily periods, over the whole sample, from 01/02/1973 to 09/24/2009 ( $\mathrm{N}=9262$ observations), and 
we compute the error for each portfolio $\gamma \in \Gamma$. We obtain

$$
\bar{E}=\frac{1}{N|\Gamma|} \sum_{\gamma \in \Gamma, n \in\{1, \ldots, N\}}\left|\bar{S}\left(k_{n}\right)-S_{n}(\gamma)\right|=2.90 \%
$$

and

$$
\breve{E}=\frac{1}{N|\Gamma|} \sum_{\gamma \in \Gamma, n \in\{1, \ldots, N\}}\left|\breve{S}\left(k_{n}\right)-S_{n}(\gamma)\right|=2.22 \%
$$

Even though $\breve{E}<\bar{E}$, the two errors are of the same order. Because $\bar{S}$ is computed under the assumptions $\left(A_{0}\right)$ and $\left(A_{1}\right)$, it has the advantage to avoid any enumeration and thus it can be used in large markets. So, in small markets $\breve{E}$ might be preferred but in large markets $\bar{E}$ is an acceptable alternative. Note that under stationarity condition and a correct choice for the distribution of the returns $\mathbf{X}$, the score $\bar{S}$ is obtained only through the computation of the parameter $k$. Thus, this score is suitable for real time applications as opposed to $\breve{S}$ which requires a complete enumeration for each realization.

\section{Comparison with the Main Performance Measures}

In this section, we assess the informativeness of our score by building a performance index based on this score and comparing its predictive ability with those of the main performance indices. The performance index in consideration is the average score of a portfolio over a training period. The measure is called average cross-section (ACS) score and the period over which the index is computed is called training period. As this measure contains cross-sectional information on the assets, it might be of some interest in order to allocate efficiently in one of investable portfolios. In Demiguel et al. (2009), the authors conclude with: "exploiting information about the cross-sectional characteristics of the assets may be a promising direction to pursue" in the design of optimal portfolios which is traditionally achieved using Sharpe-like ratios. In the following, we compare the forecasts of our measure with those obtained with the Sharpe and Sortino indices. We proceed as follows. In a given market, we first rank the LSEW portfolios according to different performance measures in a training period. Then, we study the returns of the selected portfolios after an holding period. The performance measures considered are defined as follows:

- the Sharpe ratio: for a given portfolio, we compute its in-the-sample Sharpe ratio over the training period as its average excess return (over the risk free asset) divided by the sample standard deviation of its excess returns.

- the Sortino ratio: for a given portfolio, we compute its in-the-sample Sortino ratio over the training period as its average excess return (over the risk free asset) divided by the standard 
deviation of its negative excess returns. In the case no negative excess return is observed, only those portfolios are ranked using their average excess returns.

- the ACS Score: for a given portfolio, we compute its ACS score over the training period. The ranking of this performance measure provides the highest performer in average.

Our results are obtained using two samples. The first one is the market of the 10 U.S. sectorial Datastream $^{\text {TM }}$ indices from January 1973 to May 2010 and we use the 3-month U.S. T-Bill as a risk-free rate. The second one is the market of the 10 European sectorial Datastream ${ }^{\mathrm{TM}}$ indices from November 1992 to May 2010 and we use the synthetic 3-month euro rate of Datastream as a risk-free rate. The Datastream codes of the indices and interest rates are reported in Appendix A. We consider daily, weekly and monthly frequencies in the data. For the monthly data, the periods of training are 12 and 24 months long and the holding period lasts 1 month. For the weekly data, the periods of training are 8, 16 and 24 weeks long (corresponding approximatively to 2,4 and 6 months) and the holding period lasts 1 week. For the daily data, the periods of training are 10, 20 and 40 days long (corresponding approximatively to 1, 2 and 4 months) and the holding period lasts 1 day. The results are presented below in Tables 4,5 and 6 .

The results for the monthly data are provided in Table 4. We observe that for the 12-month training period, the ACS score provides the highest and most significant returns both in the U.S. and European markets. It also provides the highest Sharpe ratio, lowest turn-over and highest ACS score. For the 24-month training period, no performance measure provides significant returns in the U.S.market while, in the European market, the Sharpe ratio provides the highest and most significant returns. It also provides the highest Sharpe ratio and the highest ACS score. We remark that, for any market and training period, the ACS score provides the lowest turn-over.

The results for the weekly data are provided in Table 5. In the U.S. market, the three measures are equivalent for the 8 -week training period. The Sharpe ratio provides the highest and most significant returns for the 16-week training period while the ACS score provides the highest and most significant returns for the 24 -week training period. In the European market, the Sortino ratio and the ACS score are tied and outperform the Sharpe ratio for the 8-week training period. The ACS score outperforms both the Sharpe and the Sortino ratio for the 16-week and 24-week training periods. As for the monthly data, the ACS score provides the lowest turn-over for any market and any training period. 


\begin{tabular}{|c|c|c|c|c|c|c|}
\hline \multicolumn{7}{|l|}{ Panel A: U.S. Market } \\
\hline Performance Measure & Training Period & Mean & (T-Stat) & Sharpe Ratio & Turn-Over & ACS Score \\
\hline Sharpe Ratio & 12 Mths & $0.2332 \%$ & $(1.98)$ & -0.3093 & $69.49 \%$ & $53.37 \%$ \\
\hline Sortino Ratio & 12 Mths & $0.1360 \%$ & $(1.06)$ & -0.4091 & $78.16 \%$ & $51.94 \%$ \\
\hline ACS Score & 12 Mths & $0.2814 \%$ & $(2.05)$ & -0.2069 & $62.30 \%$ & $54.17 \%$ \\
\hline Sharpe Ratio & 24 Mths & $0.1156 \%$ & $(0.99)$ & -0.4793 & $49.86 \%$ & $52.57 \%$ \\
\hline Sortino Ratio & 24 Mths & $0.0517 \%$ & $(0.42)$ & -0.5379 & $56.11 \%$ & $51.27 \%$ \\
\hline ACS Score & 24 Mths & $0.1361 \%$ & $(1.06)$ & -0.4068 & $41.90 \%$ & $52.43 \%$ \\
\hline \multicolumn{7}{|c|}{ Panel B: European Market } \\
\hline Performance Measure & Training Period & Mean & (T-Stat) & Sharpe Ratio & Turn-Over & ACS Score \\
\hline Sharpe Ratio & 12 Mths & $0.4170 \%$ & $(2.42)$ & 0.1571 & $71.43 \%$ & $55.43 \%$ \\
\hline Sortino Ratio & $12 \mathrm{Mths}$ & $0.5640 \%$ & $(2.95)$ & 0.3330 & $73.06 \%$ & $58.87 \%$ \\
\hline ACS Score & 12 Mths & $0.6990 \%$ & $(3.22)$ & 0.4470 & $46.12 \%$ & $59.05 \%$ \\
\hline Sharpe Ratio & 24 Mths & $0.4478 \%$ & $(2.73)$ & 0.2390 & $48.70 \%$ & $57.04 \%$ \\
\hline Sortino Ratio & 24 Mths & $0.2995 \%$ & (1.67) & 0.0083 & $50.00 \%$ & $55.65 \%$ \\
\hline ACS Score & 24 Mths & $0.3439 \%$ & $(1.57)$ & 0.0582 & $37.83 \%$ & $55.02 \%$ \\
\hline
\end{tabular}

Table 4: Basic statistics and performance measures (Sharpe ratio, Turn-Over and ACS score) of the 1-month forecasts obtained with the Sharpe ratio, the Sortino ratio and the ACS score after training periods of 12 and 24 months. This training period is the period over which the portfolio used as forecast is selected. The markets are the U.S. (panel A) and European (Panel B) markets made of the 10 sectorial Datastream indices considering monthly end-of-the-month prices. The U.S. data is from January 1973 to May 2010. The European data is from November 1992 to May 2010.

\begin{tabular}{|lr|rr|r|r|r|}
\hline Panel A: U.S. Market & \multicolumn{7}{l|}{} \\
\hline Performance Measure & Training Period & Mean & (T-Stat) & Sharpe Ratio & Turn-Over & ACS Score \\
\hline Sharpe Ratio & 8 Weeks & $0.0734 \%$ & $(3.06)$ & -0.2153 & $92.08 \%$ & $52.42 \%$ \\
Sortino Ratio & 8 Weeks & $0.0783 \%$ & $(2.58)$ & -0.1446 & $80.99 \%$ & $52.67 \%$ \\
ACS Score & 8 Weeks & $0.0711 \%$ & $(2.39)$ & -0.1878 & $69.78 \%$ & $52.74 \%$ \\
\hline Sharpe Ratio & 16 Weeks & $0.0804 \%$ & $(3.22)$ & -0.1620 & $59.47 \%$ & $52.45 \%$ \\
Sortino Ratio & 16 Weeks & $0.0458 \%$ & $(1.69)$ & -0.3596 & $62.08 \%$ & $51.88 \%$ \\
ACS Score & 16 Weeks & $0.0544 \%$ & $(1.87)$ & -0.2862 & $50.41 \%$ & $52.50 \%$ \\
\hline Sharpe Ratio & 24 Weeks & $0.0086 \%$ & $(0.34)$ & -0.6272 & $53.03 \%$ & $50.22 \%$ \\
Sortino Ratio & 24 Weeks & $0.0184 \%$ & $(0.67)$ & -0.5154 & $49.87 \%$ & $50.92 \%$ \\
ACS Score & 24 Weeks & $0.0571 \%$ & $(1.82)$ & -0.2506 & $40.73 \%$ & $52.28 \%$ \\
\hline \hline Panel B: European Market & \multicolumn{5}{|c|}{} \\
\hline Performance Measure & Training Period & Mean & $(\mathrm{T}-$ Stat) & Sharpe Ratio & Turn-Over & ACS Score \\
\hline Sharpe Ratio & 8 Weeks & $0.0556 \%$ & $(1.55)$ & -0.1315 & $94.87 \%$ & $52.19 \%$ \\
Sortino Ratio & 8 Weeks & $0.1447 \%$ & $(3.05)$ & 0.3506 & $78.50 \%$ & $54.21 \%$ \\
ACS Score & 8 Weeks & $0.1411 \%$ & $(2.98)$ & 0.3328 & $70.18 \%$ & $54.00 \%$ \\
\hline Sharpe Ratio & 16 Weeks & $0.0351 \%$ & $(0.98)$ & -0.2643 & $64.29 \%$ & $52.00 \%$ \\
Sortino Ratio & 16 Weeks & $0.1183 \%$ & $(3.13)$ & 0.2804 & $59.29 \%$ & $54.02 \%$ \\
ACS Score & 16 Weeks & $0.1494 \%$ & $(3.07)$ & 0.3718 & $48.39 \%$ & $55.31 \%$ \\
\hline Sharpe Ratio & 24 Weeks & $0.0159 \%$ & $(0.43)$ & -0.3713 & $58.20 \%$ & $51.55 \%$ \\
Sortino Ratio & 24 Weeks & $0.0589 \%$ & $(1.47)$ & -0.0872 & $50.18 \%$ & $52.71 \%$ \\
ACS Score & 24 Weeks & $0.1109 \%$ & $(2.24)$ & 0.1838 & $40.72 \%$ & $54.17 \%$ \\
\hline
\end{tabular}

Table 5: Basic statistics and performance measures (Sharpe ratio, Turn-Over and ACS score) of the 1-week forecasts obtained with the Sharpe ratio, the Sortino ratio and the ACS score after training periods of 8, 16 and 24 weeks. This training period is the period over which the portfolio used as forecast is selected. The markets are the U.S. (Panel A) and European (Panel B) markets made of the 10 sectorial Datastream indices considering weekly end-of-the-week prices. The U.S. data is from January 1973 to May 2010. The European data is from November 1992 to May 2010. 
In Table 6, we provide the results for the daily data. In the U.S. market, the ACS score provides the highest and most significant returns as well as the highest Sharpe ratio, lowest turn-over and highest ACS score for all the training periods. In the European market, we observe the same result with the ACS score outperforming the two other performance measures.

\begin{tabular}{|c|c|c|c|c|c|c|}
\hline \multicolumn{7}{|l|}{ Panel A: U.S. Market } \\
\hline Performance Measure & Training Period & Mean & (T-Stat) & Sharpe Ratio & Turn-Over & ACS Score \\
\hline Sharpe Ratio & 10 Days & $0.0217 \%$ & $(4.53)$ & -0.0078 & $81.65 \%$ & $52.30 \%$ \\
\hline Sortino Ratio & 10 Days & $0.0296 \%$ & $(5.57)$ & 0.2374 & $76.65 \%$ & $52.94 \%$ \\
\hline ACS Score & 10 Days & $0.0349 \%$ & $(6.20)$ & 0.3767 & $61.59 \%$ & $53.24 \%$ \\
\hline Sharpe Ratio & 20 Days & $0.0244 \%$ & $(5.20)$ & 0.0886 & $54.11 \%$ & $52.25 \%$ \\
\hline Sortino Ratio & 20 Days & $0.0242 \%$ & $(4.76)$ & 0.0735 & $52.88 \%$ & $52.20 \%$ \\
\hline ACS Score & 20 Days & $0.0392 \%$ & $(6.78)$ & 0.4883 & $43.46 \%$ & $53.45 \%$ \\
\hline Sharpe Ratio & 40 Days & $0.0160 \%$ & $(3.32)$ & -0.2003 & $36.63 \%$ & $51.86 \%$ \\
\hline Sortino Ratio & 40 Days & $0.0192 \%$ & $(3.75)$ & -0.0871 & $35.24 \%$ & $52.02 \%$ \\
\hline ACS Score & 40 Days & $0.0258 \%$ & $(4.40)$ & 0.1092 & $30.83 \%$ & $52.82 \%$ \\
\hline \multicolumn{7}{|c|}{$\overline{\text { Panel B: European Market }}$} \\
\hline Performance Measure & Training Period & Mean & (T-Stat) & Sharpe Ratio & Turn-Over & ACS Score \\
\hline Sharpe Ratio & 10 Days & $0.0196 \%$ & $(3.08)$ & 0.1380 & $80.71 \%$ & $51.60 \%$ \\
\hline Sortino Ratio & 10 Days & $0.0264 \%$ & $(3.54)$ & 0.3319 & $79.47 \%$ & $51.60 \%$ \\
\hline ACS Score & 10 Days & $0.0387 \%$ & $(4.41)$ & 0.6125 & $60.81 \%$ & $52.60 \%$ \\
\hline Sharpe Ratio & 20 Days & $0.0154 \%$ & $(2.40)$ & -0.0162 & $56.74 \%$ & $51.47 \%$ \\
\hline Sortino Ratio & 20 Days & $0.0203 \%$ & $(2.89)$ & 0.1515 & $53.52 \%$ & $51.57 \%$ \\
\hline ACS Score & 20 Days & $0.0314 \%$ & $(3.60)$ & 0.4202 & $44.76 \%$ & $52.47 \%$ \\
\hline Sharpe Ratio & 40 Days & $0.0235 \%$ & $(3.65)$ & 0.2877 & $38.13 \%$ & $52.04 \%$ \\
\hline Sortino Ratio & 40 Days & $0.0207 \%$ & $(2.88)$ & 0.1639 & $34.90 \%$ & $51.61 \%$ \\
\hline ACS Score & 40 Days & $0.0386 \%$ & $(4.14)$ & 0.5797 & $31.81 \%$ & $52.76 \%$ \\
\hline
\end{tabular}

Table 6: Basic statistics and performance measures (Sharpe ratio, Turn-Over and ACS score) of the 1-day forecasts obtained with the Sharpe ratio, the Sortino ratio and the ACS score after training periods of 10, 20 and 40 days. This training period is the period over which the portfolio used as forecast is selected. The markets are the U.S. (Panel A) and European (Panel B) markets made of the 10 sectorial Datastream indices considering daily prices. The U.S. data is from January 1973 to May 2010. The European data is from November 1992 to May 2010.

Over the different frequencies considered, we observe that the ACS score is the performance measure which offers the most frequently the highest and most significant returns. With the daily data, it systematically outperforms the Sharpe and Sortino ratios. It is interesting to remark that this measure has constantly provided the lowest turn-overs which means that the portfolios selected are "stable" in time and shows its ability to capture a trend.

In order to further assess the superiority of a performance measure over another one, we compare the cumulative distribution functions of the score of the different performance measures. For instance, in Figure 7, we represent the cumulative distribution functions (CDF) of the scores obtained by the portfolios selected by the Sharpe ratio, the Sortino ratio and the ACS score, in the U.S. market with a training period of 20 days. In addition, an interesting use of the score is that it allows to plot the CDF of a random allocation as well. This allocation consists in giving the same 
probability to each investable portfolio. We observe that the Sharpe and Sortino ratios dominate stochastically the random allocation while the ACS score does not. Indeed, the ACS score has the same probability to provide a portfolio with a score inferior to $20 \%$ than the random allocation. However, it also provides the highest probability to get a score higher that $50 \%$, i.e. in our case a positive return, and it clearly outperforms the Sharpe and Sortino ratios in the high scores. As a consequence, the ACS score would be prefered by risk-lover managers and the Sharpe and Sortino ratios would suit to risk-averse investors.

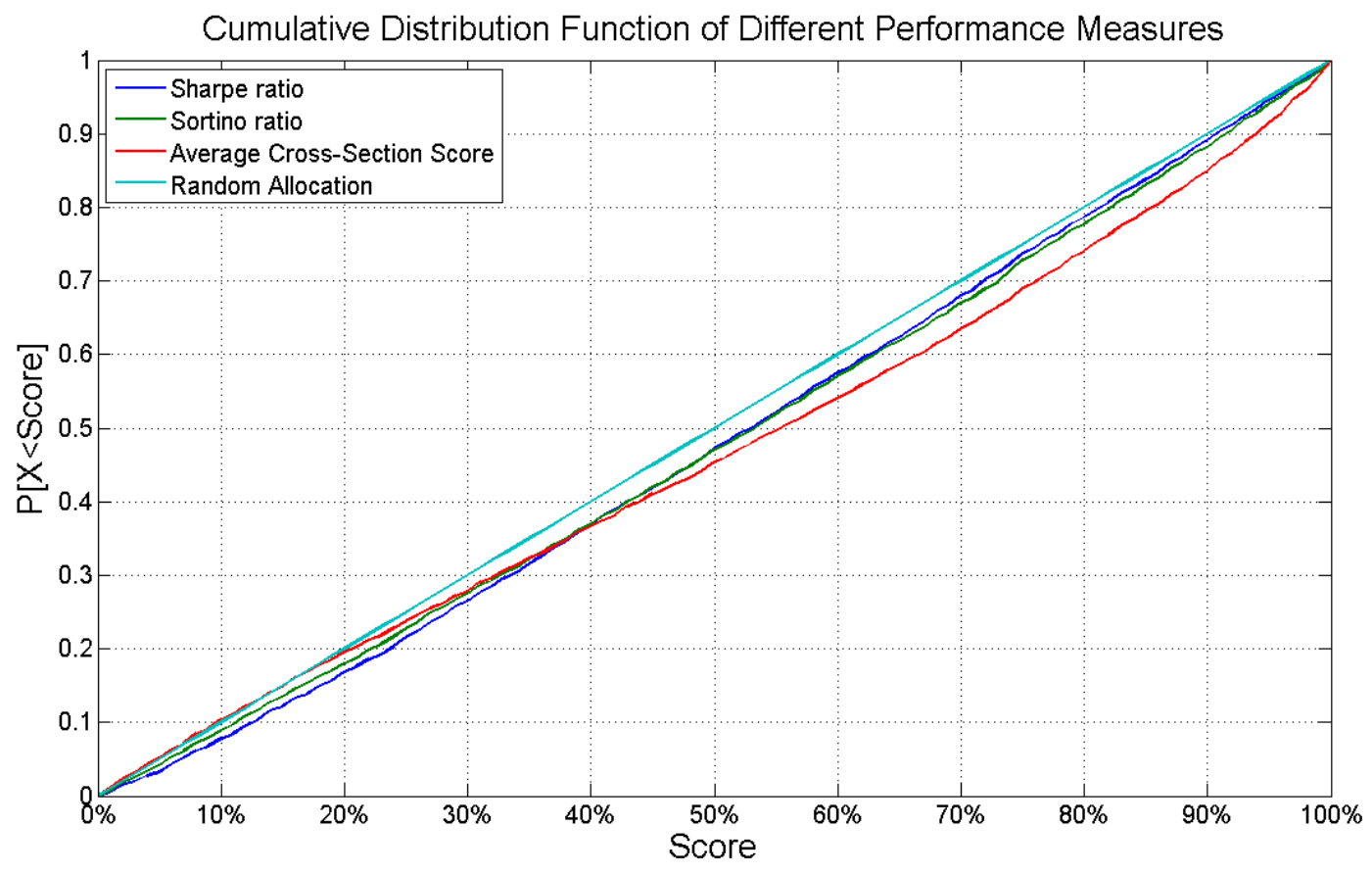

Figure 7: Cumulative Distribution Function of the scores obtained by the portfolios selected by the Sharpe ratio, the Sortino ratio, the ACS Score and a random allocation. The performance measures are used as investment method in the U.S. market with a training period of 20 days and an holding period of 1 day. The scores of the forecasts are computed by enumeration over the set of the LSEW portfolios.

Figure 8 represents the performances of the Sharpe ratio, the Sortino ratio and the ACS score in the U.S. market with a training period of 20 days and an holding period of 1 day and confirms the previous analysis. The initial investment has a value of 1 on the $01 / 02 / 1973$ and we represent the value of the investments from $01 / 02 / 1973$ to $27 / 05 / 2010$. In order to identify the effect of the recent crisis on the performances, we zoom in over the period 1992-2010 as represented in Figure 9 and we plot the crisis in shaded areas. We use the dating of the crisis proposed in Rigobon (2003) and Billio et al. (2009) which is reported in Table 7. We observe that the ACS score is the best 
performer and that it is also proner to draw-back during crisis than the two other performance measure. It is in line with the previous observation stating that the Sharpe ratio better suits a risk-averse investor than the ACS score.

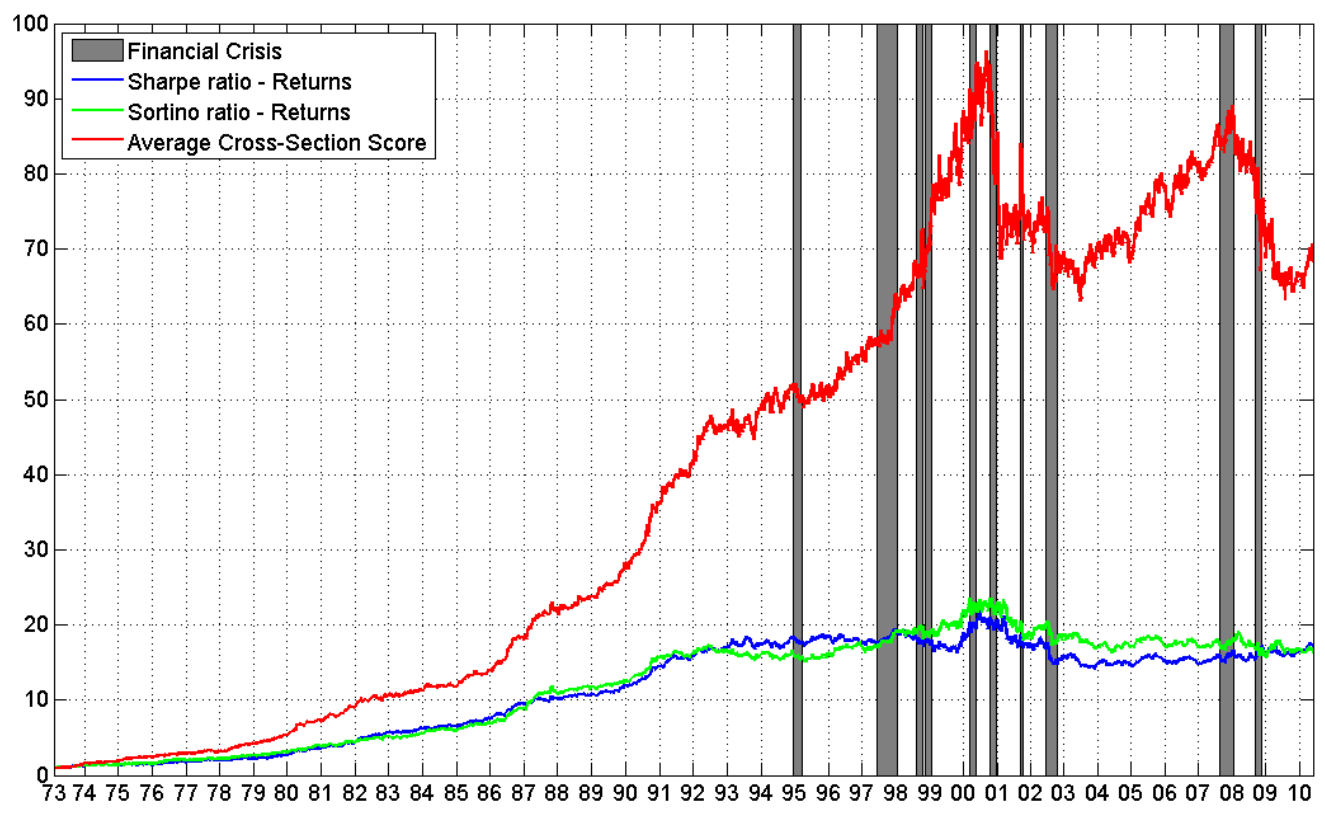

Figure 8: Performances obtained with an investment of value 1 on the 01/02/1973 investing in the forecasts obtained using the Sharpe ratio, the Sortino ratio and the ACS Score as investment method. The results presented are from the U.S. market with a training period of 20 days and an holding period of 1 day. Financial crisis are represented in shaded areas.

\begin{tabular}{|c|ll|}
\hline Crisis & Begin & End \\
\hline Mexican Crisis & December 1994 & March 1995 \\
Asian Crisis & June 1997 & January 1998 \\
Russian Crisis and LTCM & August 1998 & October 1998 \\
Brazilian Crisis & January 1999 & February 1999 \\
Internet Crash & March 2000 & May 2000 \\
Argentinean Crisis & October 2000 & December 2000 \\
September 11, 2001 & September 2001 & September 2001 \\
Worldcom, Merger dry-up & June 2002 & October 2002 \\
Mortgage Crisis & August 2007 & January 2008 \\
Global Financial Crisis & September 2008 & November 2008 \\
\hline
\end{tabular}

Table 7: Dating of the financial crisis as they appear in Figures 8 and 9.

Finally, we have seen that the new measure based on the score is globally more informative than the Sharpe and Sortino ratios for any frequency. It is proner to draw-backs but this is largely off-set by 


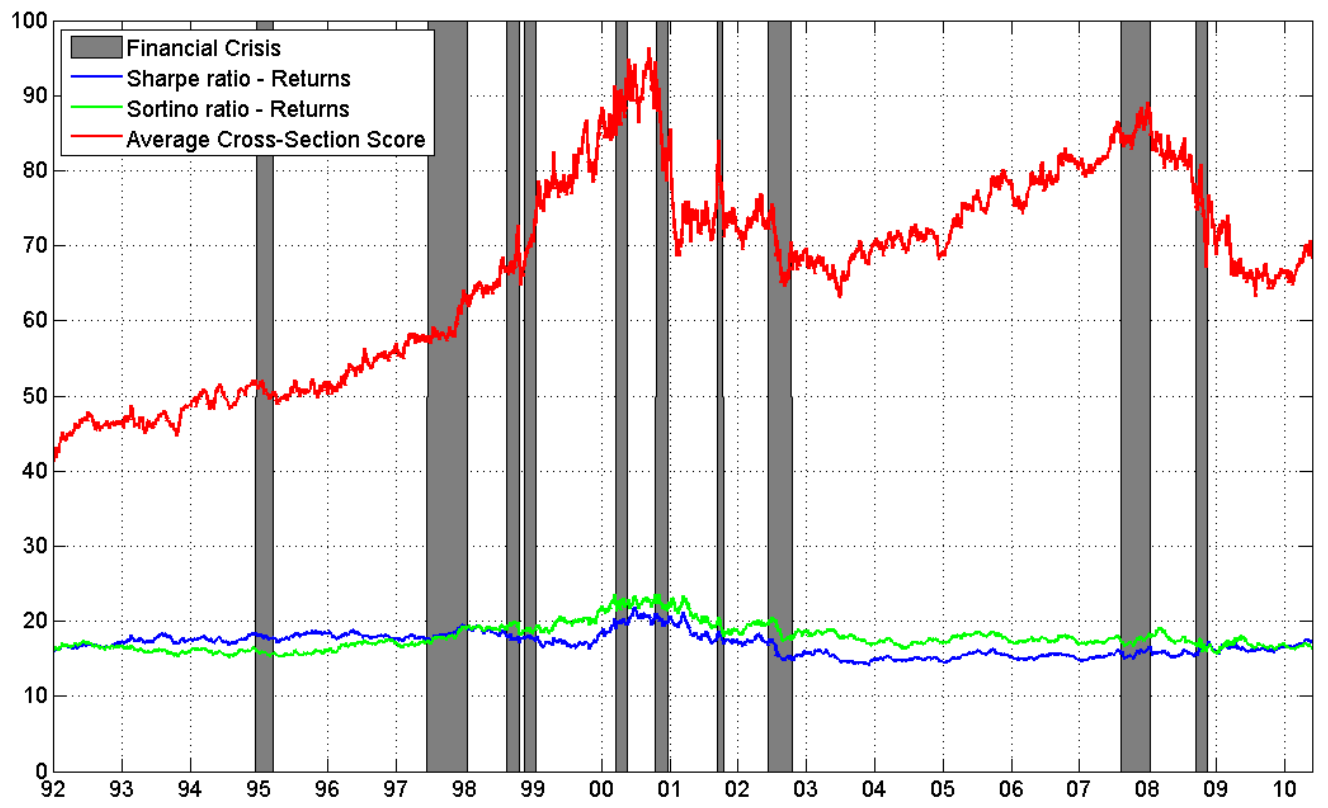

Figure 9: Zoom of Figure 8. Performances obtained with an investment of value 1 on the 01/02/1973 investing in the forecasts obtained using the Sharpe ratio, the Sortino ratio and the ACS Score as investment method. The graph focuses on the performances over the period 1992-2010. The results presented are from the U.S. market with a training period of 20 days and an holding period of 1 day. Financial crisis are represented in shaded areas.

its capacity to provide high returns as shown by the significance of its forecasts. In addition, these forecasts have a low turn-over which is interesting as a high turn-over in often a major drawback in long/short investment, Lesmond et al. (2004) and Korajczyk and Sadka (2004). These properties are very interesting for investors and encourage the study of cross-sectional effects in the markets as an alternative to the classical mean-variance optimization.

\section{Conclusion}

This paper proposes a way to quantify the goodness of an allocation through a cross-sectional score. While most of the previous works on performance measure require a peer system to appreciate a manager's performance, this approach permits to be free of this constraint. It opens new possibilities such as a nice and simple way to monitor the performance of an invested portfolio in real time and the ability for a manager to appreciate the opportunity to close his positions. In addition, it releases new information which is valuable in asset allocation. Indeed, a performance 
measure based on this score provides higher returns than the Sharpe and Sortino ratios along with a lower turnover. These results are encouraging the study of cross-sectional effects in the markets as an alternative to the classical mean-variance optimization.

\section{Appendix}

\section{A Datastream codes of the data used in this paper}

The Datastream ${ }^{\mathrm{TM}}$ codes of the World sectorial Datastream ${ }^{\mathrm{TM}}$ indices are: OILGSWD, BMATRWD, INDUSWD, CNSMGWD, HLTHCWD, CNSMSWD, TELCMWD, UTILSWD, FINANWD, TECNOWD. The Datastream ${ }^{\mathrm{TM}}$ codes of the European sectorial Datastream ${ }^{\mathrm{TM}}$ indices are: OILGSEU, BMATREU, INDUSEU, CNSMGEU, HLTHCEU, CNSMSEU, TELCMEU, UTILSEU, FINANEU, TECNOEU. The Datastream ${ }^{\mathrm{TM}}$ codes of the U.S. sectorial Datastream ${ }^{\mathrm{TM}}$ indices are: OILGSUS, BMATRUS, INDUSUS, CNSMGUS, HLTHCUS, CNSMSUS, TELCMUS, UTILSUS, FINANUS, TECNOUS. The Datastream ${ }^{\text {TM }}$ code of the 3-month U.S. T-Bill is FRTBS3M and the code the synthetic 3-month euro rate of Datastream is EMINT3M.

\section{B Proof of Proposition 1}

Let $\mathbf{X}$ be an absolutely continuous exchangeable random vector, $\mathbf{X}_{(\mathbf{n})}$ be the corresponding random vector of its order statistics. Let be $\gamma_{i} \in \Gamma$ any portfolio, $\gamma_{o}$ the optimal portfolio and $g$ the density of $\gamma_{i}^{\prime} \mathbf{X}$, then we have

$$
P\left(\gamma_{i}^{\prime} \mathbf{X}=y\right)=\sum_{\gamma \in \Gamma} P\left(\gamma^{\prime} \mathbf{X}_{(\mathbf{n})}=y\right) P\left(\gamma_{i}^{\prime} \mathbf{X}=\gamma^{\prime} \mathbf{X}_{(\mathbf{n})}\right)
$$

As $\mathbf{X}$ is an exchangeable random vector, then $\gamma$ has the same probability to be the representation of $\gamma_{i}$ in terms of order statistics, thus

$$
P\left(\gamma_{i}^{\prime} \mathbf{X}=\gamma^{\prime} \mathbf{X}_{(\mathbf{n})}\right)=\frac{1}{|\Gamma|}
$$

Plugging relationship (A-1) in expression (A-2) leads to

$$
P\left(\gamma_{i}^{\prime} \mathbf{X}=y\right)=\frac{1}{|\Gamma|} \sum_{\gamma \in \Gamma} P\left(\gamma^{\prime} \mathbf{X}_{(\mathbf{n})}=y\right) .
$$

Denoting $u_{\gamma}$ the density function of $\gamma^{\prime} \mathbf{X}_{(\mathbf{n})}$, we remark that 


$$
g=\frac{1}{|\Gamma|} \sum_{\gamma \in \Gamma} u_{\gamma}
$$

From (7), we know that if the portfolio $\gamma_{i}$ returns $k_{i}$ times the return of the optimal portfolio $\gamma_{o}$, we have

$$
\bar{S}\left(k_{i}\right)=\frac{1}{|\Gamma|} \sum_{\gamma \in \Gamma} \int_{-\infty}^{0} f_{\gamma, k_{i}}(y) d y
$$

where $f_{\gamma, k_{i}}$ is the density function of $\left(\gamma-k_{i} \tilde{\gamma}_{o}\right)^{\prime} \mathbf{X}_{(\mathbf{n})}$. Denoting $h_{k_{i}}$ the density of $-k_{i} \tilde{\gamma}_{o}^{\prime} \mathbf{X}_{(\mathbf{n})}$, we obtain

$$
\bar{S}\left(k_{i}\right)=\frac{1}{|\Gamma|} \int_{-\infty}^{0} \sum_{\gamma \in \Gamma}\left(u_{\gamma} * h_{k_{i}}\right)(y) d y .
$$

Using the property of distributivity of the convolution product, the relationship (A-6) can be rewritten as follows:

$$
\bar{S}\left(k_{i}\right)=\frac{1}{|\Gamma|} \int_{-\infty}^{0}\left(\left(\sum_{\gamma \in \Gamma} u_{\gamma}\right) * h_{k_{i}}\right)(y) d y
$$

Now, from (A-4), we have $\sum_{\gamma \in \Gamma} u_{\gamma}=|\Gamma| g$, and the relationship (A-7) becomes:

$$
\begin{aligned}
\bar{S}(k) & =\frac{1}{|\Gamma|} \int_{-\infty}^{0}\left(|\Gamma| g * h_{k_{i}}\right)(y) d y \\
& =\int_{-\infty}^{0}\left(g * h_{k_{i}}\right)(y) d y .
\end{aligned}
$$

The proof of Proposition 1 is complete.

\section{Proof of Proposition 2}

Let $\mathbf{X}=\left(X_{1}, X_{2}, \ldots, X_{n}\right)^{\prime}$ be an absolutely continuous exchangeable random vector distributed according to the multivariate generalized hyperbolic distribution $G H_{n}(\lambda, \chi, \psi, \mu, \Sigma, \kappa), \mathbf{X}_{(\mathbf{n})}$ be the random vector of its order statistics, $\gamma \in \Gamma$ be a portfolio and $\gamma_{o}$ the optimal portfolio. In Proposition 1, we established that $\bar{S}$ depends on $\left(g * h_{k}\right)$. Here, we need to study separately $g$, the 
distribution of $\gamma^{\prime} \mathbf{X}$, and $h_{k}$, the distribution of $\tilde{\gamma}_{o}^{\prime} \mathbf{X}_{(\mathbf{n})}$. We begin with the study of $g$ in Corollary 1 .

Corollary 1: Let $\mathbf{X}=\left(X_{1}, X_{2}, \ldots, X_{n}\right)^{\prime}$ be an absolutely continuous exchangeable random vector distributed according to the multivariate generalized hyperbolic distribution $G H_{n}(\lambda, \chi, \psi, \mu, \Sigma, \kappa)$ and $\gamma \in \Gamma$ be any LSEW portfolio, then $\gamma^{\prime} \mathbf{X}$ is distributed according to an elliptically contoured distribution such that

$$
\gamma \mathbf{X} \sim E C_{1}\left(0, \sigma^{2}(1-\rho) \frac{4}{n}, \phi^{(1)}\right)
$$

where the density generator $\phi^{(1)}$ is given by

$$
\phi^{(1)}(u)=C_{1} \frac{K_{\lambda-\frac{1}{2}}(\sqrt{\psi(\chi+u)})}{(\sqrt{\chi+u})^{\frac{1}{2}-\lambda}}
$$

with $C_{1}$ a normalizing constant and $K_{\nu}$ the modified Bessel function of the third kind.

Proof of Corollary 1:

From McNeil et al. (2005), we know that the generalized hyperbolic distributions are closed under linear transformation. So, if $\mathbf{X} \sim G H_{n}(\lambda, \chi, \psi, \mu, \Sigma, \kappa)$ and $\mathbf{Y}=\gamma^{\prime} \mathbf{X}$ where $\gamma \in R^{n}$, then

$$
\mathbf{Y} \sim G H_{1}\left(\lambda, \chi, \psi, \gamma^{\prime} \mu, \gamma^{\prime} \Sigma \gamma^{\prime}, \gamma^{\prime} \kappa\right)
$$

In our case, we have

- $\gamma$ is a LSEW portfolio, so $\gamma^{\prime} \mathbf{1}_{\mathbf{n}}=0$, thus $\gamma^{\prime} \mu=0$ and $\gamma^{\prime} \kappa=0$

- the random variables are exchangeable, so $\Sigma=\sigma^{2}\left[(1-\rho) I_{n}+\rho \mathbf{1}_{\mathbf{n}} \mathbf{1}_{\mathbf{n}}{ }^{\prime}\right]$, where $\sigma$ is the scale and $\rho$ is the correlation

Consequently, $\gamma^{\prime} \mathbf{X}$ is distributed as follows

$$
\gamma^{\prime} \mathbf{X} \sim G H_{1}\left(\lambda, \chi, \psi, 0, \sigma^{2}(1-\rho) \frac{4}{n}, 0\right)
$$


i.e. $\gamma^{\prime} \mathbf{X}$ follows a symmetric generalized hyperbolic distribution.

From Schmidt (2003) (p.54, definition 3.2.12), we know that the symmetric generalized hyperbolic distribution $G H_{n}(\lambda, \chi, \psi, \mu, \Sigma, 0)$ is the elliptically contoured distribution $E C_{n}(\mu, \Sigma, \phi)$ where the density generator $\phi^{(n)}$ is given by

$$
\phi^{(n)}(u)=C_{n} \frac{K_{\lambda-\frac{n}{2}}(\sqrt{\psi(\chi+u)})}{(\sqrt{\chi+u})^{\frac{n}{2}-\lambda}}
$$

with $C_{n}$ a normalizing constant defined in Schmidt (2003) (formula 5.3) and $K_{\nu}$ the modified Bessel function of the third kind. So, in our case, we have

$$
\gamma^{\prime} \mathbf{X} \sim E C_{1}\left(0, \sigma^{2}(1-\rho) \frac{4}{n}, \phi^{(1)}\right)
$$

where

$$
\phi^{(1)}(u)=C_{1} \frac{K_{\lambda-\frac{1}{2}}(\sqrt{\psi(\chi+u))}}{(\sqrt{\chi+u})^{\frac{1}{2}-\lambda}} .
$$

The proof of Corollary 1 is complete.

Now, we investigate the distribution of $\tilde{\gamma}_{o}^{\prime} \mathbf{X}_{(\mathbf{n})}$ :

Corollary 2: Let $\mathbf{X}=\left(X_{1}, X_{2}, \ldots, X_{n}\right)^{\prime}$ be an absolutely continuous exchangeable random vector distributed according to the multivariate generalized hyperbolic distribution $G H_{n}(\lambda, \chi, \psi, \mu, \Sigma, \kappa)$, $\mathbf{X}_{(\mathbf{n})}$ be the random vector of its order statistics and $\tilde{\gamma}_{o} \in \Gamma$ be the order statistics representation of the optimal portfolio, then $\tilde{\gamma}_{o}^{\prime} \mathbf{X}_{(\mathbf{n})}$ is distributed according to an elliptically contoured distribution such that

$$
\tilde{\gamma}_{o}^{\prime} \mathbf{X}_{(\mathbf{n})}={ }^{d} \sigma \sqrt{1-\rho} \tilde{\gamma}_{o}^{\prime} \mathbf{U}_{(\mathbf{n})}
$$

where $\rho \in[0,1)$ and $\mathbf{U}_{(\mathbf{n})}$ is the vector of order statistics induced by the spherically contoured 
random vector $\mathbf{U} \sim E C_{n}\left(\mathbf{0}, I_{n}, \phi^{(n)}\right)$ with $\phi^{(n)}$ given by

$$
\phi^{(n)}(u)=C_{n} \frac{K_{\lambda-\frac{n}{2}}(\sqrt{\psi(\chi+u))}}{(\sqrt{\chi+u})^{\frac{n}{2}-\lambda}}
$$

with $C_{n}$ a normalizing constant and $K_{\nu}$ the modified Bessel function of the third kind.

Proof of Corollary 2:

From Arellano-Valle and Genton (2007) (Corollary 1), we have

$$
{\tilde{\gamma_{o}}}^{\prime} \mathbf{X}_{(\mathbf{n})}{ }^{d}\left(\tilde{\gamma}_{o}{ }^{\prime} \mathbf{X} \mid \Delta \mathbf{X} \geq \mathbf{0}\right)
$$

where $\Delta$ is such that $\boldsymbol{\Delta} \mathbf{X}=\left(X_{2}-X_{1}, X_{3}-X_{2}, \ldots, X_{n}-X_{n-1}\right)^{\prime}$. We note that $\Delta \Delta^{\prime}=\left(\delta_{i, j}\right), \delta$ being the Kronecker product, with $\delta_{i, i}=2, \delta_{i-1, i}=\delta_{i+1, i}=-1$ and $\delta_{i, j}=0$ otherwise. The generalized hyperbolic distributions are closed under linear transformation and $\mathbf{X}$ is an exchangeable random vector, so we have

$$
\Delta \mathbf{X} \sim G H_{n-1}\left(\lambda, \chi, \psi, 0, \sigma^{2}(1-\rho) \Delta \Delta^{\prime}, 0\right)
$$

Thus, from Schmidt (2003) as seen in Corollary 1, $\Delta \mathbf{X}$ follows an elliptically contoured distribution

$$
\boldsymbol{\Delta} \mathbf{X} \sim E C_{n-1}\left(0, \sigma^{2}(1-\rho) \Delta \Delta^{\prime}, \phi^{(n-1)}\right)
$$

where

$$
\phi^{(n-1)}(u)=C_{n-1} \frac{K_{\lambda-\frac{n-1}{2}}(\sqrt{\psi(\chi+u)})}{(\sqrt{\chi+u})^{\frac{n-1}{2}-\lambda}} .
$$

Since $\tilde{\gamma}_{o}$ is a LSEW portfolio, relationship (B-6) holds. So, from expression (B-6) and expression (B-12), we have

$$
\left\{\begin{array}{l}
\tilde{\gamma}_{o}^{\prime} \mathbf{X} \sim E C_{1}\left(0, \sigma^{2}(1-\rho) \frac{4}{n}, \phi^{(1)}\right) \\
\Delta \mathbf{X} \sim E C_{n-1}\left(0, \sigma^{2}(1-\rho) \Delta \Delta^{\prime}, \phi^{(n-1)}\right)
\end{array}\right.
$$

which are the intermediary results obtained in the proof of Corollary 3 in Arellano-Valle and Genton 
(2007). Thus, Corollary 3 can be used here, and we extend it to generalized hyperbolic distributions. It follows

$$
\tilde{\gamma}_{o}^{\prime} \mathbf{X}_{(\mathbf{n})}={ }^{d} \sigma \sqrt{1-\rho} \tilde{\gamma}_{o}^{\prime} \mathbf{U}_{(\mathbf{n})}
$$

where $\mathbf{U}_{(\mathbf{n})}$ is the vector of order statistics induced by the spherically contoured random vector $\mathbf{U} \sim E C_{n}\left(\mathbf{0}, I_{n}, \phi^{(n)}\right)$ and $\rho \in[0,1)$.

The proof of Corollary 2 is complete.

Now, we prove Proposition 2. From Corollary 1 and denoting $Z \sim E C_{1}\left(0, \frac{4}{n}, \phi^{(1)}\right)$, we have

$$
\gamma^{\prime} \mathbf{X}={ }^{d} \sigma \sqrt{1-\rho} Z
$$

Then, from Corollary 2 and relationship (B-16), we have

$$
\gamma^{\prime} \mathbf{X}-k \tilde{\gamma}_{o}^{\prime} \mathbf{X}_{(\mathbf{n})}={ }^{d} \sigma \sqrt{1-\rho}\left(Z-k \tilde{\gamma}_{o}^{\prime} \mathbf{U}_{(\mathbf{n})}\right)
$$

Let denote $v_{k}$ the density function of $Z-k{\tilde{\gamma_{o}}}^{\prime} \mathbf{U}_{(\mathbf{n})}$. From (B-17), we have the following expression of $\bar{S}(k)$ :

$$
\bar{S}(k)=\int_{-\infty}^{0}\left(g * h_{k}\right)(y) d y=\int_{-\infty}^{0} v_{k}(y) d y
$$

So, $\bar{S}(k)$ is independent of $\mu, \sigma, \rho$ and $\kappa$.

The proof of Proposition 2 is complete.

\section{References}

Arellano-Valle, R., and M. Genton. "On the exact distribution of linear combinations of order statistics from dependent random variables." Journal of Multivariate Analysis, 97 (2007) 18761894

Barndorff-Nielsen, O.E. "Exponentially decreasing distributions for the logarithm of particle size." Proceedings of the Royal Society of London A, 353 (1977) 401-419 
Barndorff-Nielsen, O.E., J. Kent; and M. Sørensen. "Normal variance-mean mixture and zdistributions." International Statistical Review, 50 (1982) 145-159

Billio, M., L. Calès and D. Guégan. "Portfolio Symmetry and Momentum." Working Paper WP.2009.03 Centre d'Économie de la Sorbonne, Paris, France (2009)

Billio, M., M. Getmansky and L. Pelizzon. "Crises and Hedge Fund Risk." Working Paper 10-08 Università Ca'Foscari, Venice, Italy (2009)

Brandt, M., P. Santa-Clara and R. Valkanov. "Parametric Portfolio Policies: Exploiting Charateristics in the Cross-Section of Equity Returns." Review of Financial Studies, 22 (2009), 3411-3447

Chan, K., H. Alaudeen and T. Wilson. "Profitability of momentum strategies in the international equity markets." Journal of Financial and Quantitative Analysis, 35 (2000) 153-172

Christie, S.. "Beware the Sharpe ratio." Macquarie Applied Finance Centre, Macquarie University, Sydney, Australia

Darolles, S., C. Gourieroux and J. Jasiak. "L-performance with an application to hedge funds." Journal of Empirical Finance, 16 (2009) 671-685

Demiguel, V., L. Garlappi and R. Uppal. "Optimal versus Naive Diversification: How inefficient is the 1/N Portfolio Strategy." The Review of Financial Studies, 22 (2009) 1915-1953

Eberlein, E., and U. Keller. "Hyperbolic Distributions in Finance.” Bernoulli, 1 (1995) 281-299

Fajardo, J., and A. Farias. "Multivariate affine generalized hyperbolic distributions: an empirical investigation." International Review of Financial Analysis, 18 (2009) 174-184

Gatev, E., W. Goetzmann and G. Rouweunhorst. "Pairs trading: performance of a relative value arbitrage rule." Working Paper 7032, National Bureau of Economic Research, United States (1999)

Grinblatt, M., and S. Titman. "A study of monthly mutual funds returns and performance evaluation techniques." Journal of Financial and Quantitative Analysis, 29 (1994) 419-444

Hadar, J., and W. Russel. "Rules for Ordering Uncertain Prospects." American Economic Review, 59 (1969) 25-34

Jeegadeesh, N., and S. Titman. "Returns to buying winners and selling losers: Implications for stock market efficiency." Journal of Finance, 48 (1993) 65-91

Jensen, M. "The performance of mutual funds in the period 1945-1964." Journal of Business, 42 (1968) 167-247 
Kazemi, H., T. Schneeweis and R. Spurgin. "Momentum in asset returns: are commodity returns a special case ?" Journal of Alternative Investments, 10 (2009) 23-36

Korajczyk R. and R. Sadka. "Are Momentum Profits Robust to Trading Costs?" Journal of Finance, 59-3 (2004) 1039-1082

Lesmond D., Schill M. and C. Zhou. "The illusory nature of momentum profits." Journal of Financial Economics, 71 (2004) 349-380

Lo, A. "The Statistics of Sharpe Ratios", Financial Analysts Journal, July/August (2002) 36-52

McNeil, A., R. Frey and P. Embrechts. "Quantitative Risk Management: Concepts, Techniques and Tools." Princeton University Press (2005).

Okunev, K. and D. White. "Do momentum-based strategies still work in foreign currency markets ?" Journal of Financial and Quantitative Analysis, 38 (2003) 425-447

Prause, K. "The Generalized Hyperbolic Model: Estimation, Financial Derivatives and Risk Measures." Ph.D. Dissertation. University of Freiburg, Germany (1999)

Quirk, J. and R. Saposnik. "Admissibility and Measurable Utility Functions." Review of Economic Studies, 29 (1962) 140-146

Rigobon, R. "Identification Through Heteroskedasticity." Journal of Political Economy, 11 (2003) $642-685$

Rouwenhorst, K. "International Momentum Strategies." Journal of Finance, 53 (1998) 267-284

Schmidt, R. "Dependencies of Extreme Events in Finance." Ph.D. Dissertation. University of Ulm, Germany (2003) http://stats.lse.ac.uk/schmidt

Shadwick, W. and C. Keating "A Universal performance measure." Journal of Performance Measure, Spring (2002) 59-84.

Sharpe, W. "Mutual fund performance." Journal of Business, 39 (1966) 119-138

Sharpe, W. "The Sharpe Ratio.” Journal of Portfolio Management, 21 (1994) 49-58

Treynor, J. "How to rate management of investment funds." Harvard Business Review, 43 (1965) $63-75$ 